\title{
The Bethe-Goldstone Equation with Singular Interactions: A Fully Off-Shell Solution for the Boundary Condition Model
}

\author{
R. F. BISHOP \\ Department of Theoretical Physics, University of Manchester, Manchester M13 9PL, England; and \\ Daresbury Laboratory, Daresbury, Warrington WA4 4AD, England
}

Received May 1, 1975

\begin{abstract}
The mutual interaction of a pair of fermions imbedded in a many-body system of identical particles when they are excited out of the filled Fermi sea, is studied via the $T$-matrix or transition amplitude specified by the Bethe-Goldstone (BG) equation. The role of the bare two-body interaction is emphasised, and in particular the consequences are elucidated of whether the potential is "well-behaved" (nonsingular) or not. The properties of the BG $T$-matrix, including generalized orthonormality and completeness relations, are derived both for nonsingular potentials and for singular potentials containing an infinite hard core. General analytic properties are exploited to derive relations that express the fully off-shell BG $T$-matrix purely in terms of the half-shell amplitude (and the properties of any possible bound states in the medium). The general formalism is illustrated by deriving exact analytic expressions for the fully off-shell BG $T$-matrices for a pair of particles with equal and opposite momenta interacting via either of two singular model interactions; namely, the pure hard-core interaction and the boundary condition model. Results for both models are expressed in terms of the solution to a simple one-dimensional Fredholm integral equation. The analytic properties of the solutions are discussed and exploited to prove both their uniqueness and that they satisfy the various general relations derived. To our knowledge, these results represent the first exact nontriviai solution to the fully off-shell BG equation for any local potential, or singular limiting case thereof.
\end{abstract}

\section{INTRODUCTION}

In most realistic many-body problems the interparticle interactions are either too strong or too singular to permit straightforward perturbation expansions in powers of the potential. Typically the two-body interaction becomes strong and repulsive at short range, ensuring that a series expansion for any physical quantity in powers of the potential will at best converge only very slowly. Brueckner [1] was the first to realize within the many-body context that this case could be treated mathematically by rearranging into a single term each of the separate terms in the original perturbation series that involve successive interactions between the same pair of particles. 
Within the framework of time-independent perturbation theory Goldstone's theorem [2] re-expresses the full perturbation sum for the ground-state energy of a many-body system in terms of the so-called connected terms only. This series in turn can be rearranged so that the two-body potential is replaced by the transition operator or $T$-matrix that exactly sums to all orders the repeated interactions between the same two particles (the ladder diagrams). Just as the LippmannSchwinger equation [3] for the $t$-matrix describes the scattering of a pair of particles in vacuo, so the Bethe-Goldstone [4] equation for the $T$-matrix describes the analogous situation for the pair imbedded in a many-body medium of identical particles. The aim of the present paper is to study some general properties of the Bethe-Goldstone (BG) $T$-matrix for both well-behaved and singular interactions, and to calculate exactly the fully off-shell BG $T$-matrix for some simple model interactions, namely, the pure hard core (HC) interaction, and its generalization to the pure boundary condition model (BCM) [5].

A study of the BG T-matrix (or its equivalent) is useful for two main reasons. It both describes the effect of the many-body medium on the two-body interactions and correlations; and it serves as a sufficient (and for singular potentials, necessary) precursor for calculations of the thermodynamic functions of the many-body system. For dilute systems, the $T$-matrix alone is sufficient for a complete description whereas for denser systems where many-particle cluster contributions are not negligible the two-body $T$-matrix may profitably be used as the input, as in the evaluation of the three-body cluster terms considered by Bethe and his collaborators [6] using the techniques of Faddeev [7].

There are also excellent reasons for explicitly studying the HC interaction or the BCM in this context. On the one hand, both models are analytically very simple, and their free scattering $t$-matrices are known in exact closed form [8]. On the other hand, many realistic potentials exist in nature which contain strong shortrange repulsion which is often well approximated by the $\mathrm{HC}$ or the $\mathrm{BCM}$, and by adding suitable attractive tails external to these singular models one can often obtain excellent quantitative agreement with experiment. Thus, for example, several excellent analytic fits to the two-nucleon data exist which contain a hard core $[9,10]$ or which are based on the BCM [11]. It is our hope that the exact results obtained in this paper can profitably be employed in such systems. Also, exact results in many-body theory are rare, and can often be used as a yardstick against which to measure more elaborate calculations, and to obtain exact properties. For example, all calculations to date dealing with the ground-state energy of a system of hard-sphere fermions in powers of the hard-sphere diameter $a$ (see, e.g., [12]) have found nonphysical logarithmic divergences, which arise from using a series expansion for the $T$-matrix rather than its exact form.

The basic plan of the present work is that general properties of the BG $T$-matrix are first developed, placing particular emphasis on the differences that arise 
between singular and non-singular interactions. The general techniques are then applied to the $\mathrm{HC}$ and BCM, and exact analytic expressions are obtained in each case for the fully off-shell BG $T$-matrices, for the physically most interesting case where the interacting pair has zero total momentum. Furthermore, the uniqueness of each solution (expressed in each partial wave as the solution to a simple one-dimensional Fredholm integral equation) is explicitly demonstrated. To the knowledge of the present author, this work presents the first exact solution for a fully off-shell BG $T$-matrix for any two-body interaction, apart from the basically trivial known result for a separable potential.

\section{Bethe-Goldstone Equation}

The Bethe-Goldstone (BG) $T$-matrix is the scattering function which describes the transition of a pair of particles from an initial to a final state when they are imbedded in a many-fermion system of identical particles, and when the pair interact together an arbitrary number of times via their bare two-body potential. During the transition, the many-body background, which is at zero temperature and in its groundstate, is considered to be inert; and it affects the transition only through the Pauli principle by restricting the available intermediate states. The BG equation [4] is most readily written in terms of the relative and average momenta of the scattering pair, defined in terms of their initial and final momenta $\mathbf{p}_{1}, \mathbf{p}_{2}$ and $\mathbf{p}_{1}{ }^{\prime}, \mathbf{p}_{2}{ }^{\prime} ;$ respectively, as

$$
\begin{aligned}
& \mathbf{p}=\frac{1}{2}\left(\mathbf{p}_{1}-\mathbf{p}_{2}\right), \quad \mathbf{p}^{\prime}=\frac{1}{2}\left(\mathbf{p}_{1}{ }^{\prime}-\mathbf{p}_{2}{ }^{\prime}\right) ; \\
& \mathbf{P}=\frac{1}{2}\left(\mathbf{p}_{1}+\mathbf{p}_{2}\right)=\frac{1}{2}\left(\mathbf{p}_{1}{ }^{\prime}+\mathbf{p}_{2}{ }^{\prime}\right) .
\end{aligned}
$$

The BG equation for the fully-off-shell $T$-matrix then takes the form,

$$
\begin{aligned}
& T\left(\mathbf{p}, \mathbf{p}^{\prime} ; s+i \eta, \mathbf{P}\right) \\
& \quad=u\left(\mathbf{p}-\mathbf{p}^{\prime}\right)-\int \frac{d \mathbf{q}}{(2 \pi)^{3}} u(\mathbf{p}-\mathbf{q}) \frac{Q(\mathbf{q}, \mathbf{P})}{q^{2}-s-i \eta} T\left(\mathbf{q}, \mathbf{p}^{\prime} ; s+i \eta, \mathbf{P}\right),
\end{aligned}
$$

where

$$
V(\mathbf{r})=m^{-1} u(\mathbf{r})=m^{-1}(2 \pi)^{-3} \int d \mathbf{p} e^{-i \mathbf{p} \cdot \mathbf{r}} u(\mathbf{p}),
$$

is the two-particle potential, and $m$ is the mass of each particle. The quantity $s / m$ is the total energy of the scattering pair in the CM frame, and the $T$-matrix is said to be fully-off-shell for those values of its arguments for which the inequalities $p^{2} \neq s \neq p^{\prime 2}$ hold. The factor $\eta$ in Eq. (2.2) is a positive infinitesimal, and is a stylised reminder of the outgoing-wave boundary condition at infinity which we 
impose on the scattering pair. The function $Q(\mathbf{q}, \mathbf{P})$ in Eq. (2.2) which restrains the particles to lie outside the Fermi sea in intermediate scattering states, has the form

$$
Q(\mathbf{q}, \mathbf{P})=\theta\left(|\mathbf{P}+\mathbf{q}|-k_{F}\right) \theta\left(|\mathbf{P}-\mathbf{q}|-k_{F}\right),
$$

where $k_{F}$ is the Fermi momentum, and $\theta(x)$ the unit-step function,

$$
\begin{aligned}
\theta(x) & =1, & & x>0 \\
& =0, & & x<0 .
\end{aligned}
$$

For many purposes it is convenient to extend the domain of $s$ from being a real variable to the entire complex plane. Where permissible this analytic continuation is performed by the substitution $s+i \eta \rightarrow z$ in Eq. (2.2). It is also useful to write Eq. (2.2) as an operator equation, fre from a particular representation, as

$$
\begin{aligned}
T(z, \mathbf{P}) & =u-u Q(\mathbf{P}) g_{0}(z) T(z, \mathbf{P}) \\
& =u-T(z, \mathbf{P}) Q(\mathbf{P}) g_{0}(z) u
\end{aligned}
$$

where it is easily shown that Eq. (2.5b) has the same solution as Eq. (2.5a), assuming that this latter equation has a unique solution. In the relative momentum representation, the various operators in Eqs. (2.5) have matrix elements given by,

$$
\begin{aligned}
\left\langle\mathbf{p}|T(z, \mathbf{P})| \mathbf{p}^{\prime}\right\rangle & =T\left(\mathbf{p}, \mathbf{p}^{\prime} ; z, \mathbf{P}\right), \\
\left\langle\mathbf{p}|u| \mathbf{p}^{\prime}\right\rangle & =u\left(\mathbf{p}-\mathbf{p}^{\prime}\right), \\
\left\langle\mathbf{p}|Q(\mathbf{P})| \mathbf{p}^{\prime}\right\rangle & =(2 \pi)^{3} \delta\left(\mathbf{p}-\mathbf{p}^{\prime}\right) Q(\mathbf{p}, \mathbf{P}), \\
\left\langle\mathbf{p}\left|g_{0}(z)\right| \mathbf{p}^{\prime}\right\rangle & =(2 \pi)^{3} \delta\left(\mathbf{p}-\mathbf{p}^{\prime}\right)\left(p^{2}-z\right)^{-1} ;
\end{aligned}
$$

and operator multiplication is defined by

$$
\left\langle\mathbf{p}|A B| \mathbf{p}^{\prime}\right\rangle=(2 \pi)^{-3} \int d \mathbf{q}\langle\mathbf{p}|A| \mathbf{q}\rangle\left\langle\mathbf{q}|B| \mathbf{p}^{\prime}\right\rangle .
$$

The operator $g_{0}(s+i \eta)$ is just the free two-particle propagator with the outgoingwave boundary condition at infinity. Evidently the operators $g_{0}{ }^{\dagger}$ and $T^{\dagger}$ are appropriate to the corresponding incoming-wave boundary condition. It is apparent that in the limit as $k_{F} \rightarrow 0$, which corresponds to the many-body background becoming infinitely dilute, the operator $Q(\mathbf{P})$ can be replaced by unity; and the BG $T$-matrix reduces to the free scattering $t$-matrix, which satisfies the LippmannSchwinger equation,

$$
\begin{aligned}
t(z) & =u-u g_{0}(z) t(z) \\
& =u-t(z) g_{0}(z) u .
\end{aligned}
$$


The potential operator $u$ is readily eliminated between Eqs. (2.5) and (2.7) to give the relation,

$$
\begin{gathered}
T(z, \mathbf{P})=t(z)+t(z) \bar{Q}(\mathbf{P}) g_{0}(z) T(z, \mathbf{P}) \\
\bar{Q}(\mathbf{P})=1-Q(\mathbf{P}) .
\end{gathered}
$$

This last equation is particularly suitable for solution of the BG $T$-matrix in cases where the potential is singular, since the potential has now been subsumed into the free $t$-matrix which is often a well-behaved function. In particular, we shall study Eq. (2.8) in Sections 5 and 6 for the case of the hard-core potential, and in Section 7 for the boundary-condition model.

The obvious first step in solving either Eq. (2.5) or (2.8) is to make a partial-wave decomposition in the variable $\cos \theta=\hat{p} \cdot \hat{p}^{\prime}$, in the relative momentum representation. Unfortunately, the dependence of the $T$-matrix on the total momentum $\mathbf{P}$ of the interacting pair, serves to destroy spherical symmetry, and neither integral equation decouples in partial waves (although by parity conservation, the odd and even partial waves separately decouple). We shall therefore only study the $T$-matrix for zero total momentum, $\mathbf{P}=0$, of the pair, which for many cases is the situation of most physical interest. Defining the partial-wave decomposition of the general function $Z\left(\mathbf{p}, \mathbf{p}^{\prime} ; s\right)$ by

$$
Z\left(\mathbf{p}, \mathbf{p}^{\prime} ; s\right)=\sum_{l}(2 l+1) Z_{l}\left(p, p^{\prime} ; s\right) P_{l}\left(\hat{p} \cdot \hat{p}^{\prime}\right),
$$

Eq. (2.8) reduces in the case $\mathbf{P}=0$, to

$$
\begin{aligned}
& T_{l}\left(p, p^{\prime} ; s+i \eta\right) \\
& \quad=t_{l}\left(p, p^{\prime} ; s+i \eta\right)+\int_{0}^{k_{F}} \frac{q^{2} d q}{2 \pi^{2}} \frac{t_{l}(p, q ; s+i \eta) T_{l}\left(q, p^{\prime} ; s+i \eta\right)}{q^{2}-s-i \eta},
\end{aligned}
$$

where we have introduced the notation

$$
T\left(\mathbf{p}, \mathbf{p}^{\prime} ; s+i \eta, \mathbf{P}=0\right) \equiv T\left(\mathbf{p}, \mathbf{p}^{\prime} ; s+i \eta\right) .
$$

For many purposes it is convenient to introduce a generalized fully off-shell BG wavefunction operator, $X(z, \mathbf{P})$, defined in terms of the BG $T$-matrix operator as

$$
T(z, \mathbf{P})=u X(z, \mathbf{P}) ;
$$

and whose matrix elements in the relative momentum representation are written as

$$
\left\langle\mathbf{p}|X(z, \mathbf{P})| \mathbf{p}^{\prime}\right\rangle=X\left(\mathbf{p}, \mathbf{p}^{\prime} ; z, \mathbf{P}\right) .
$$

The case of free (i.e., in vacuo, where $Q \rightarrow 1$ ) two-particle scattering is similarly described by a free wavefunction operator $\chi(z)$. Using Eqs. (2.5) and (2.7) together 
with these definitions, it is clear that these generalized wavefunction operators can be written in terms of their respective transition operators as,

$$
\begin{aligned}
X(z, \mathbf{P}) & =1-Q(\mathbf{P}) g_{0}(z) T(z, \mathbf{P}), \\
\chi(z) & =1-g_{0}(z) t(z),
\end{aligned}
$$

in which expressions the potential operator $u$ has been eliminated. From these generalized wavefunction operators, one can define the usual half-off-shell wavefunctions as

$$
\begin{aligned}
\Psi_{\mathbf{p}^{\prime}}^{(\mathbf{P})}(\mathbf{p}) & =X\left(\mathbf{p}, \mathbf{p}^{\prime} ; p^{\prime 2}+i \eta, \mathbf{P}\right) \\
\psi_{\mathbf{p}^{\prime}}(\mathbf{p}) & =\chi\left(\mathbf{p}, \mathbf{p}^{\prime} ; p^{\prime 2}+i \eta\right) .
\end{aligned}
$$

Making use of Eqs. (2.12), we have the relations

$$
\begin{gathered}
\Psi_{\mathbf{p}^{\prime}}^{(\mathbf{p})}(\mathbf{p})=(2 \pi)^{3} \delta\left(\mathbf{p}-\mathbf{p}^{\prime}\right)-\frac{Q(\mathbf{p}, \mathbf{P}) F\left(\mathbf{p}, \mathbf{p}^{\prime} ; \mathbf{P}\right)}{p^{2}-p^{\prime 2}-i \eta}, \\
\psi_{\mathbf{p}^{\prime}}(\mathbf{p})=(2 \pi)^{3} \delta\left(\mathbf{p}-\mathbf{p}^{\prime}\right)-\frac{\tilde{f}\left(\mathbf{p}, \mathbf{p}^{\prime}\right)}{p^{2}-p^{\prime 2}-i \eta},
\end{gathered}
$$

for the BG and free half-shell wavefunctions in terms of the respective half-shell transition functions,

$$
\begin{aligned}
F\left(\mathbf{p}, \mathbf{p}^{\prime} ; \mathbf{P}\right) & =T\left(\mathbf{p}, \mathbf{p}^{\prime} ; p^{\prime 2}+i \eta, \mathbf{P}\right) \\
\tilde{f}\left(\mathbf{p}, \mathbf{p}^{\prime}\right) & =t\left(\mathbf{p}, \mathbf{p}^{\prime} ; p^{\prime 2}+i \eta\right) .
\end{aligned}
$$

Note that with our choice of normalization, the fully on-shell free $t$-matrix in the $l$ th partial wave, is given in terms of the phase-shift $\delta_{l}$, by

$$
\tilde{f}_{l}(p, p)=-4 \pi p^{-1} e^{i \delta_{l}(p)} \sin \delta_{l}(p) .
$$

The aim of the remainder of this work is to investigate the general properties of the BG $T$-matrix for both nonsingular and singular potentials, and to solve Eq. (2.9) for the particular singular cases corresponding to the pure hard-core interaction and the pure $\mathrm{BCM}$.

\section{General Properties of the BG $T$-Matrix}

In this Section we shall derive some general properties of the BG $T$-matrix, working directly from its definition in Eq. (2.5), and we shall particularly indicate the differences that arise when the potential is allowed to be singular. To be specific we shall work only with the case $\mathbf{P}=0$ from now on. 


\subsection{Hermitian Analyticity}

We shall only be interested from the outset in cases where the inter-particle potential is hermitian, viz., $u=u^{\dagger}$. For such a hermitian potential, it is easy to show from Eq. $(2.5 \mathrm{~b})$ that $T\left(z^{*}\right)$ satisfies

$$
T\left(z^{*}\right)=u-T\left(z^{*}\right) Q g_{0}\left(z^{*}\right) u .
$$

Similarly, the hermitian conjugate of Eq. (2.5a) leads to

$$
T^{\dagger}(z)=u-T^{\dagger}(z) Q g_{0}^{\dagger}(z) u .
$$

Since $g_{0}\left(z^{*}\right)=g_{0}^{\dagger}(z)$ from the definition of Eq. (2.6), it is clear from comparison of Eqs. (3.1) and (3.2) that if Eq. (2.5) has a unique solution, then $T(z)$ is a hermitian analytic operator, viz., it satisfies the relation

$$
T^{\dagger}(z)=T\left(z^{*}\right) .
$$

Taking matrix elements of this relation gives equivalently

$$
T^{*}\left(\mathbf{p}^{\prime}, \mathbf{p} ; z\right)=T\left(\mathbf{p}, \mathbf{p}^{\prime} ; z^{*}\right) .
$$

\subsection{Time-Reversal Invariance and Symmetry}

By taking matrix elements of Eq. (2.5) it is easy to show that the $T$-matrix is unchanged under time-reversal, viz,

$$
T\left(\mathbf{p}, \mathbf{p}^{\prime} ; z\right)=T\left(-\mathbf{p}^{\prime},-\mathbf{p} ; z\right) .
$$

However, since $T\left(\mathbf{p}, \mathbf{p}^{\prime} ; z\right)$ can only be a function of the variables $p^{2}, p^{\prime 2}, \mathbf{p} \cdot \mathbf{p}^{\prime}$ and $z$ by rotational invariance, this relation can be equivalently written as the symmetry relation

$$
T\left(\mathbf{p}, \mathbf{p}^{\prime} ; z\right)=T\left(\mathbf{p}^{\prime}, \mathbf{p} ; z\right),
$$

which together with Eq. (3.3b) leads us to the relation

$$
T\left(\mathbf{p}, \mathbf{p}^{\prime} ; z^{*}\right)=T^{*}\left(\mathbf{p}, \mathbf{p}^{\prime} ; z\right) .
$$

Hence $T\left(\mathbf{p}, \mathbf{p}^{\prime} ; z\right)$ is a real analytic function of the variable $z$.

\subsection{Generalized Unitarity}

It is an easy matter to eliminate the potential operator $u$ between Eqs. (2.5a) and (3.2) to give the relation (3.7a),

$$
\begin{aligned}
T(z)-T^{\dagger}(z) & =T^{\dagger}(z) Q\left[g_{0}^{\dagger}(z)-g_{0}(z)\right] T(z) \\
& =T(z) Q\left[g_{0}^{\dagger}(z)-g_{0}(z)\right] T^{\dagger}(z) .
\end{aligned}
$$


Equation (3.7b) is derived similarly by eliminating $u$ from Eq. (2.5b) and its hermitian conjugate. By putting $z=s+i \eta$ in this result, and taking the partialwave decomposition leads easily to the relation

$$
\text { Im } \begin{aligned}
T_{l}\left(p, p^{\prime} ; s+i \eta\right) & =(2 i)^{-1}\left[T_{l}\left(p, p^{\prime} ; s+i \eta\right)-T_{l}\left(p, p^{\prime} ; s-i \eta\right)\right] \\
& =-(4 \pi)^{-1} \kappa \theta\left(\kappa-k_{F}\right) F_{l}^{*}(p, \kappa) F_{l}\left(p^{\prime}, \kappa\right) \\
& =-(4 \pi)^{-1} \kappa \theta\left(\kappa-k_{F}\right) F_{l}(p, \kappa) F_{l}^{*}\left(p^{\prime}, \kappa\right),
\end{aligned}
$$

for $s>0$ and $\kappa=+s^{1 / 2}$, and where we have used Eqs. (3.5) and (3.6). Considering the quantity $T_{l}\left(p, p^{\prime} ; z\right)$ as a function of the complex energy parameter $z$, Eq. (3.8) shows that $T_{l}$ has a cut along the right-hand real axis in the range $\left(k_{F}{ }^{2}, \infty\right)$, and the discontinuity across the cut is expressible purely in terms of the half-shell amplitudes $F_{l}\left(p, p^{\prime}\right)$.

\subsection{A Generalized Orthonormality Relation}

By taking matrix elements between the states $\langle\mathbf{p}|$ and $\left|\mathbf{p}^{\prime}\right\rangle$ of both Eqs. (2.5a) and (2.5b) and setting $z=p^{\prime 2}+i \eta$ in the former, one again has two equations between which the potential $u\left(\mathbf{p}-\mathbf{p}^{\prime}\right)$ can be eliminated. Performing this calculation and decomposing the resulting relation in partial waves readily leads to the result

$$
\begin{aligned}
& T_{l}\left(p, p^{\prime} ; z\right)-F_{l}\left(p, p^{\prime}\right) \\
& \quad=\int_{k_{F}}^{\infty} \frac{q^{2} d q}{2 \pi^{2}} T_{l}(p, q ; z)\left[\frac{1}{q^{2}-p^{\prime 2}-i \eta}-\frac{1}{q^{2}-z}\right] F_{l}\left(q, p^{\prime}\right) .
\end{aligned}
$$

A particularly useful form of this equation can be obtained by putting $z=p^{2}-i \eta$, and using Eqs. (3.3b) and (2.15a) to write the result as

$$
\frac{F_{l}\left(p, p^{\prime}\right)-F_{l}^{*}\left(p^{\prime}, p\right)}{p^{2}-p^{\prime 2}-i \eta}=\int_{k_{F}}^{\infty} \frac{q^{2}}{2} \frac{d q}{\pi^{2}} \frac{F_{l}^{*}(q, p) F_{l}\left(q, p^{\prime}\right)}{\left(q^{2}-p^{2}+i \eta\right)\left(q^{2}-p^{\prime 2}-i \eta\right)} .
$$

Defining an operator $J$ by its matrix elements in the momentum space representation of the plane-wave states $|\mathbf{p}\rangle$ by

$$
\left\langle\mathbf{p}|J| \mathbf{p}^{\prime}\right\rangle \equiv \frac{F\left(\mathbf{p}, \mathbf{p}^{\prime}\right)}{p^{2}-p^{\prime 2}-i \eta},
$$

the previous relation (3.10) can be equivalently written as an operator equation,

$$
J+J^{\dagger}=J^{\dagger} Q J
$$

This last equation can be written more suggestively in terms of the BG wavefunction,

$$
\Psi_{\mathbf{p}^{\prime}}^{(\mathbf{p}=0)}(\mathbf{p})=\Psi_{\mathbf{p}^{\prime}}(\mathbf{p})=\left\langle\mathbf{p} \mid \Psi_{\mathbf{p}^{\prime}}\right\rangle
$$


which from Eq. (2.14a) is given by

$$
\left|\Psi_{\mathbf{p}^{\prime}}\right\rangle=(1-Q J)\left|\mathbf{p}^{\prime}\right\rangle .
$$

Using Eq. (3.12) it is an easy matter to show that the overlap of two BG wavefunctions can be expressed as

$$
\begin{aligned}
\left\langle\Psi_{\mathbf{k}} \mid \Psi_{\mathbf{k}^{\prime}}\right\rangle & \equiv(2 \pi)^{-3} \int d \mathbf{q} \Psi_{\mathbf{k}}^{*}(\mathbf{q}) \Psi_{\mathbf{k}^{\prime}}(\mathbf{q}) \\
& =\left\langle\mathbf{k}\left|1+\bar{Q} J+J^{+} \bar{Q}\right| \mathbf{k}^{\prime}\right\rangle \\
& =(2 \pi)^{3} \delta\left(\mathbf{k}-\mathbf{k}^{\prime}\right)+\frac{\theta\left(k_{F}-k\right) F\left(\mathbf{k}, \mathbf{k}^{\prime}\right)-\theta\left(k_{F}-k^{\prime}\right) F^{*}\left(\mathbf{k}^{\prime}, \mathbf{k}\right)}{k^{2}-k^{\prime 2}-i \eta} .
\end{aligned}
$$

This relation is a generalized orthonormality relation for the BG wavefunctions $\left|\Psi_{\mathbf{k}}\right\rangle$, which are thus seen to form an orthonormal set only for $k>k_{F}$.

\subsection{Analyticity and a Generalized Completeness Relation}

In Section 3.3 it was demonstrated that the function $T_{l}\left(p, p^{\prime} ; z\right)$ considered as a function of the complex energy parameter $z$ has a quasi-unitarity cut along the right-hand real axis, with a branch-point threshold at $k_{F}{ }^{2}$; and the discontinuity across the cut was evaluated in terms of the half-shell quantity $F_{l}\left(p, p^{\prime}\right)$. Let us assume for the moment that $T_{l}\left(p, p^{\prime} ; z\right)$ has no other singularities in the complex $z$-plane. By analogy with free potential scattering, it is to be expected that this statement is synonymous with the assumption that the potential sustains no bound BG pairs in the many-body medium. With this assumption, and a knowledge of the behavior of $T_{l}\left(p, p^{\prime} ; z\right)$ as $|z| \rightarrow \infty$, it is possible to write a dispersion relation for $T_{l}$ in the parameter $z$. We do this by considering the contour integrals defined as

$$
E_{n}(z) \equiv \frac{1}{2 \pi i} \oint_{C} d z^{\prime} \frac{f_{n}\left(z^{\prime}\right)}{z^{\prime}-z},
$$

for the three cases

$$
\begin{aligned}
& f_{0}(z)=T_{l}\left(p, p^{\prime} ; z\right), \\
& f_{1}(z)=\left(z-p^{\prime 2}-i \eta\right)^{-1} T_{l}\left(p, p^{\prime} ; z\right), \\
& f_{2}(z)=\left(z-p^{2}-i \eta\right)^{-1}\left(z-p^{\prime 2}-i \eta\right)^{-1} T_{l}\left(p, p^{\prime} ; z\right),
\end{aligned}
$$

where the contour $C$ is as shown in Fig. 1, in the limit that the radius $R$ of the large circular arc tends to infinity. The integrals $E_{n}(z)$ will converge only if the functions $f_{n}(z)$ behave suitably at infinity; or more precisely it is required that

$$
z^{-n-n} f_{n}(z) \underset{|z| \rightarrow \infty}{\longrightarrow} 0 ; \quad \eta>0 .
$$




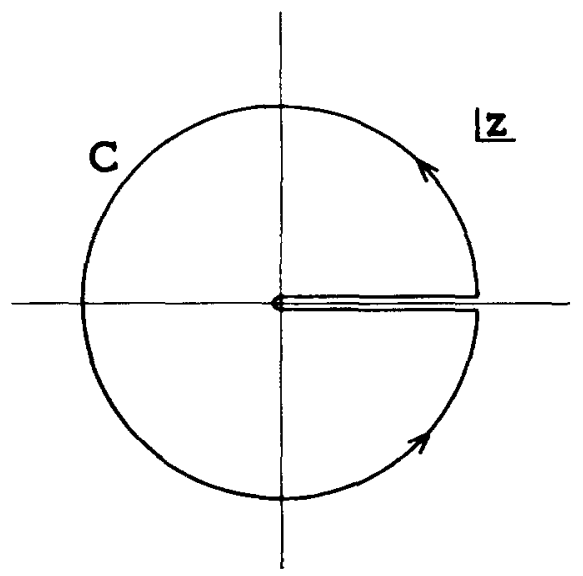

FiG, 1. The contour $C$ in the complex $z$-plane.

In the parlance of dispersion theory, the integrals $E_{n}$ for the cases $n=0,1,2$ thus correspond respectively to unsubtracted, once-subtracted and twice-subtracted dispersion integrals for the function $T_{l}\left(p, p^{\prime} ; z\right)$. It is with regard to this point that we are careful to distinguish two cases of particular interest, viz, (a) where the two-particle potential is nonsingular (NS); and (b) where the two-particle potential is of the singular hard core (SHC) type. In this context an NS potential is reckoned to be an ordinary well-behaved potential such that the Fourier transforms $u_{l}\left(p, p^{t}\right)$ exist. In this case it is clear by inspection of the BG $T$-matrix Eq. (2.2) that the behavior of the $T$-matrix at large values of $|z|$ is given by ${ }^{1}$

$$
T_{l}\left(p, p^{\prime} ; z\right) \underset{|z| \rightarrow \infty}{\longrightarrow} u_{l}\left(p, p^{\prime}\right) \text {. }
$$

In contrast, potentials of the singular type do not have well-defined Fourier transforms, although the free $t$-matrix remains well-behaved. In this case the BG $T$-matrix is defined to be the solution to Eq. (2.9), where the inhomogeneous term is the free $t$-matrix appropriate to the SHC potential. For the case of SHC potentials the free $t$-matrix has the asymptotic form

$$
t_{l}\left(p, p^{\prime} ; z\right) \underset{i z \mid \rightarrow \infty}{\longrightarrow}-z \theta_{l}\left(p, p^{\prime}\right)
$$

${ }^{1}$ Henceforth in this Section we adopt the convention of labelling equations with the letter $N$ or $S$ after the equation number to indicate relations that are valid only for nonsingular or singular potentials of the hard-core type, respectively. An equation number containing ncither letter is to be considered valid in general. 
where $\theta_{l}\left(p, p^{\prime}\right)$ is a well-behaved function defined explicitly in Section 4, and direct inspection of Eq. (2.9) gives the asymptotic result,

$$
T_{l}\left(p, p^{\prime} ; z\right) \underset{|z| \rightarrow \infty}{\longrightarrow}-z \Theta_{l}\left(p, p^{\prime}\right),
$$

where the function $\Theta_{l}\left(p, p^{\prime}\right)$ satisfies the integral equation,

$$
\Theta_{l}\left(p, p^{\prime}\right)=\theta_{l}\left(p, p^{\prime}\right)+\left(2 \pi^{2}\right)^{-1} \int_{0}^{h_{F}} q^{2} d q \theta_{l}(p, q) \Theta_{l}\left(q, p^{\prime}\right) .
$$

We shall use Eq. (3.17S) as a definition of what is meant by an SHC interaction potential.

Using these results on the asymptotic behaviour of the BG $T$-matrix and the previous assumptions on its analytic properties, it is an easy matter to evaluate the integrals $E_{n}(z)$ in two distinct ways, viz, by use of Cauchy's theorem, and by direct integration around the contour $C$. The three cases $n=0,1,2$ in Eq. (3.15) are now dealt with in turn.

(i) $n=0$. In this case the integral $E_{0}(z)$ exists only for NS potentials, since from Eq. (3.18S) it is easy to see that the contribution from the infinite circular arc in the case of SHC interactions is unbounded. Thus, dealing only with NS potentials, simple use of Cauchy's theorem leads immediately to the result

$$
E_{0}(z)=T_{l}\left(p, p^{\prime} ; z\right),
$$

since by assumption the function $T_{l}\left(p, p^{\prime} ; z\right)$ has no singularities inside the contour $C$. By direct integration around the contour, we have

$$
\begin{aligned}
E_{0}(z)= & \frac{1}{2 \pi i} \lim _{R \rightarrow \infty} \int_{0}^{2 \pi} \frac{\operatorname{Re}^{i \theta} i d \theta}{\operatorname{Re}^{i \theta}-z} T_{l}\left(p, p^{\prime} ; \operatorname{Re}^{i \theta}\right) \\
& +\frac{1}{2 \pi i} \int_{k_{F^{2}}}^{\infty} d k^{2} \frac{\left[T_{l}\left(p, p^{\prime} ; k^{2}+i \eta\right)-T_{l}\left(p, p^{\prime} ; k^{2}-i \eta\right)\right]}{k^{2}-z} .
\end{aligned}
$$

Comparison of these two expressions leads immediately to the relation,

$$
T_{l}\left(p, p^{\prime} ; z\right)=u_{l}\left(p, p^{\prime}\right)-\int_{k_{F}}^{\infty} \frac{q^{2} d q}{2 \pi^{2}} \frac{F_{l}(p, q) F_{l}^{*}\left(p^{\prime}, q\right)}{q^{2}-z}
$$

where we have used both Eq. (3.16N) and the unitarity relation (3.8).

(ii) $n=1$. The integral $E_{1}(z)$ exists for both NS and SHC potentials and we can evaluate both exactly as above. The only difference between the NS and SHC results is that the contribution to the contour integral from the infinite circular 
arc vanishes in the former case, but remains finite in the latter. In the same manner as before, we obtain the two results,

$$
\begin{aligned}
\frac{T_{l}\left(p, p^{\prime} ; z\right)-F_{l}\left(p, p^{\prime}\right)}{p^{\prime 2}-z+i \eta} & =\int_{k_{F}}^{\infty} \frac{q^{2} d q}{2 \pi^{2}} \frac{F_{l}(p, q) F_{l}^{*}\left(p^{\prime}, q\right)}{\left(q^{2}-z\right)\left(q^{2}-p^{\prime 2}-i \eta\right)} \\
& =\int_{k_{F}}^{\infty} \frac{q^{2} d q}{2 \pi^{2}} \frac{F_{l}(p, q) F_{l}^{*}\left(p^{\prime}, q\right)}{\left(q^{2}-z\right)\left(q^{2}-p^{\prime 2}-i \eta\right)}+\Theta_{l}\left(p, p^{\prime}\right)
\end{aligned}
$$

In the case of NS potentials, Eq. (3.21N) is just a once-subtracted form of the previous Eq. (3.20N), whereas for SHC interactions, Eq. (3.21S) is a new relation. A particularly useful form of Eqs. (3.21) is obtained by putting $z=p^{2}-i \eta$. In operator notation, the resulting relations can be written as

$$
\begin{aligned}
J+J^{\dagger} & =J Q J^{\dagger} \\
& =J Q J^{\dagger}+\Theta
\end{aligned}
$$

which should be compared with Eq. (3.12). These last operator identities can in turn be cast as an equation for the BG wavefunctions using Eq. (3.13). It is an easy matter to obtain the result,

$$
\begin{aligned}
\int_{k_{F}}^{\infty} \frac{d \mathbf{k}}{(2 \pi)^{3}} \Psi_{\mathbf{k}}(\mathbf{p}) \Psi_{\mathbf{k}}{ }^{*}\left(\mathbf{p}^{\prime}\right) & \\
=\theta\left(p-k_{F}\right) \theta\left(p^{\prime}-k_{F}\right) & \times(2 \pi)^{3} \delta\left(\mathbf{p}-\mathbf{p}^{\prime}\right) \\
& \times\left[(2 \pi)^{3} \delta\left(\mathbf{p}-\mathbf{p}^{\prime}\right)-\Theta\left(\mathbf{p}, \mathbf{p}^{\prime}\right)\right],
\end{aligned}
$$

or equivalently in operator notation,

$$
\begin{aligned}
\left|\Psi_{\mathbf{k}}\right\rangle\left\langle\mathbf{k}|Q| \mathbf{k}^{\prime}\right\rangle\left\langle\Psi_{\mathbf{k}^{\prime}}\right| & =Q \\
& =Q(1-\Theta) Q
\end{aligned}
$$

where the integrations over the variables $\mathbf{k}$ and $\mathbf{k}^{\prime}$ are understood. These generalized completeness relations show that for NS potentials, the BG wavefunctions $\Psi_{\mathbf{k}}(\mathbf{p})$ form a complete set in the range $p>k_{F}$ only, whereas for SHC potentials the BG wavefunctions are never complete. The operator $\Theta$, which is a measure of the incompleteness of the set, compares exactly with the operator $\theta$ for the free scattering wavefunctions for an SHC potential. This lack of completeness is a reflection of the fact that the BG wavefunctions $\Psi_{\mathrm{k}}(\mathbf{r})$ are identically zero for $r<a$ just as are the free scattering wavefunctions from an SHC potential. It is also worth pointing out that the completeness or lack of completeness of the wavefunctions (for 
relative momentum $p>k_{F}$ ) is determined directly from the asymptotic behavior of $T_{l}\left(p, p^{\prime} ; z\right)$ as $|z| \rightarrow \infty$.

(iii) $n=2$. The integral $E_{2}(z)$ of Eq. (3.15) can be evaluated exactly as before, and in this case the contribution to the contour integral from the infinite circular arc is identically zero for both NS and SHC potentials. Performing the integrations as above leads to the result,

$$
\begin{aligned}
T_{l}\left(p, p^{\prime} ; z\right)= & \frac{1}{\left(p^{\prime 2}-p^{2}\right)}\left[\left(p^{\prime 2}-z\right) F_{l}\left(p^{\prime}, p\right)-\left(p^{2}-z\right) F_{l}\left(p, p^{\prime}\right)\right. \\
& -\left(p^{2}-z\right)\left(p^{\prime 2}-z\right) \int_{k_{F}}^{\infty} \frac{k^{2} d k}{2 \pi^{2}} \frac{F_{l}(p, k) F_{l}^{*}\left(p^{\prime}, k\right)}{k^{2}-z} \\
& \left.\times\left(\frac{1}{k^{2}-p^{\prime 2}-i \eta}-\frac{1}{k^{2}-p^{2}-i \eta}\right)\right]
\end{aligned}
$$

valid for both NS and SHC potentials, and in which form $T_{l}\left(p, p^{\prime} ; z\right)$ is manifestly a symmetric function of its first two arguments.

Up to this point we have made the assumption that the only singularity of $T_{l}\left(p, p^{\prime} ; z\right)$ in the complex $z$-plane is the right-hand cut. It is self-evident how to modify the various relations derived from the dispersion integrals $E_{n}(z)$ if the functions $T_{l}$ also possess simple poles on the left-hand real axis at the positions $z=-m \hbar^{-2}\left|E_{i}^{(l)}\right|$ which will correspond to bound BG pairs in the medium, the $i$ th bound-state pair in the lth partial-wave channel having a binding energy $E_{i}^{(l)}$. Since we shall mainly be interested in the remainder of this work with application to the repulsive pure hard-core (HC) interaction, we have chosen to assume the absence of bound-state poles, and any other singularities in the BG $T$-matrix. Using this assumption it is clear that the fully off-shell BG $T$-matrix is fully determined from the half-shell quantity for either NS or SHC interactions by the use of any of Eqs. (3.20), (3.21), or (3.25); and in particular for the pure $\mathrm{HC}$ interaction, Eq. (3.25) would be the obvious choice. In fact we shall choose not to use Eq. (3.25) to evaluate $T_{l}\left(p, p^{\prime} ; z\right)$ for the pure $\mathrm{HC}$ interaction, but shall solve Eq. (2.9) directly for this case, in order to gain a deeper insight into the problem. Finally after obtaining the solution, we shall verify that it obeys the various general rclations derived in this Section. In particular it will be shown to satisfy Eqs. (3.21S) and (3.25); thereby justifying our assumptions made in their derivation for this example.

To fulfill this plan, the free scattering $t$-matrix for the pure $\mathrm{HC}$ interaction, which is used as an input for the solution, is first reviewed in Section 4. The halfshell BG $T$-matrix is then derived in Section 5 , and the derivation is extended to the fully off-shell situation in Section 6. 


\section{Free-Scattering $t$-Matrix for the Pure HC Interaction}

In a previous paper [8], the fully off-shell $t$-matrix has been derived for free (i.e., in vacuo) scattering with a pure hard-core (HC) interparticle potential of spatial extent $a$,

$$
\begin{aligned}
V(r) & =\infty, & & r<a \\
& =0, & & r>a .
\end{aligned}
$$

The salient features and the detailed expression for the $\mathrm{HC} t$-matrix are reviewed in this Section. The fully off-shell HC $t$-matrix can be expressed in terms of the half-shell transition amplitude $\tilde{f}_{l}\left(p, p^{\prime}\right)$ as

$$
t_{l}\left(p, p^{\prime} ; s+i \eta\right)=\left(p^{\prime 2}-s\right) \theta_{l}\left(p, p^{\prime}\right)+b_{l}\left(p^{\prime}, \kappa\right) \tilde{f}_{l}(p, \kappa)
$$

where $\kappa \equiv s^{1 / 2}$, and

$$
\begin{aligned}
b_{l}(p, \kappa) & =i \kappa\left(p^{2}-\kappa^{2}\right) \int_{a}^{\infty} r^{2} d r j_{l}(p r) h_{l}^{+}(\kappa r) \\
& =i \kappa a^{2}\left[p j_{l}^{\prime}(p a) h_{l}^{+}(\kappa a)-\kappa j_{l}(p a) h_{l}^{+\prime}(\kappa a)\right] .
\end{aligned}
$$

The functions $j_{l}(x)$ and $n_{l}(x)$ are spherical Bessel functions of the first and second kind, and

$$
h_{l}^{ \pm}(x)=j_{l}(x) \pm \operatorname{in}_{l}(x)
$$

are the corresponding spherical Hankel functions. The notation

$$
f^{\prime}(p a)=\left.(d / d x) f(x)\right|_{x=p a},
$$

is employed in Eq. (4.2). Use of the Wronskian relation

$$
j_{l}(x) n_{l}{ }^{\prime}(x)-j_{l}^{\prime}(x) n_{l}(x)=x^{-2},
$$

easily leads to the relation

$$
b_{l}(p, p)=1 \text {. }
$$

The function $\theta_{l}\left(p, p^{\prime}\right)$ in Eq. (4.1) is the Fourier transform of the unit-step function $\theta(a-r)$ in coordinate-space, in the lth partial wave,

$$
\begin{aligned}
\theta_{l}\left(p, p^{\prime}\right) & =4 \pi \int_{0}^{a} r^{2} d r j_{l}(p r) j_{l}\left(p^{\prime} r\right) \\
& =\left(4 \pi a^{2} /\left(p^{\prime 2}-p^{2}\right)\right)\left[p j_{l}\left(p^{\prime} a\right) j_{l}(p a)-p^{\prime} j_{l}(p a) j_{l}^{\prime}\left(p^{\prime} a\right)\right]
\end{aligned}
$$


Putting $\kappa=p^{\prime}$ in Eq. (4.1), and using Eq. (4.4), identifies $\tilde{f}_{l}\left(p, p^{\prime}\right)$ as the half-shell $t$-matrix, which in turn has the form

$$
\tilde{f}_{l}\left(p, p^{\prime}\right)=t_{l}\left(p, p^{\prime} ; p^{\prime 2}+i \eta\right)=4 \pi j_{l}(p a) / i p^{\prime} h_{l}{ }^{+}\left(p^{\prime} a\right) .
$$

The $t$-matrix $t_{l}\left(p, p^{\prime} ; s+i \eta\right)$ is symmetric between its arguments $p$ and $p^{\prime}$ as a consequence of time-reversal invariance [cf. Eq. (3.5)], and the form given in Eq. (4.1) is easily manipulated into the manifestly symmetric form,

$$
\begin{aligned}
& t_{l}\left(p, p^{\prime} ; s+i \eta\right) \\
& \quad=\frac{1}{\left(p^{\prime 2}-p^{2}\right)}\left[\left(p^{\prime 2}-s\right) b_{l}(p, \kappa) \tilde{f}_{l}\left(p^{\prime}, \kappa\right)-\left(p^{2}-s\right) b_{l}\left(p^{\prime}, \kappa\right) \tilde{f}_{l}(p, \kappa)\right] .
\end{aligned}
$$

In turn, this equation can be rewritten by eliminating the functions $b_{l}$ by making use of the relation which follows from Eq. (4.1) by the replacement $\kappa=p$, to give

$$
\begin{aligned}
t_{l}\left(p, p^{\prime} ; s+i \eta\right)= & {\left[\left(\left(p^{2}-s\right)\left(p^{\prime 2}-s\right) /\left(p^{2}-p^{2}\right)\right)\left\{\tilde{f}_{l}(p, \kappa) \theta_{l}\left(p^{\prime}, \kappa\right)-\tilde{f}_{l}\left(p^{\prime}, \kappa\right) \theta_{l}(p, \kappa)\right\}\right.} \\
& \left.+\tilde{f}_{l}(p, \kappa) \tilde{f}_{l}\left(p^{\prime}, \kappa\right)\right]\left(1 / \tilde{f}_{l}(\kappa, \kappa)\right) .
\end{aligned}
$$

It will prove to be convenient for future use to introduce the notation,

$$
\begin{aligned}
d_{l}(p) & \equiv 4 \pi a^{2} j_{l}(p a), \\
c_{l}(p) & =p j_{l}^{\prime}(p a), \\
m_{l}(p) & \equiv\left[i p a^{2} h_{l}^{+}(p a)\right]^{-1} ;
\end{aligned}
$$

in terms of which we may write

$$
\begin{aligned}
\tilde{f}_{l}\left(p, p^{\prime}\right) & =d_{l}(p) m_{l}\left(p^{\prime}\right), \\
\theta_{l}\left(p, p^{\prime}\right) & =\left[d_{l}\left(p^{\prime}\right) c_{l}(p)-c_{l}\left(p^{\prime}\right) d_{l}(p)\right] /\left(p^{\prime 2}-p^{2}\right), \\
b_{l}\left(p, p^{\prime}\right) m_{l}\left(p^{\prime}\right) & =c_{l}(p)+d_{l}(p)\left[m_{l}\left(p^{\prime}\right)-c_{l}\left(p^{\prime}\right)\right] / d_{l}\left(p^{\prime}\right) ;
\end{aligned}
$$

where the last relation follows from Eq. (4.2) by using the Wronskian relation (4.3).

Perhaps the most important aspect of the HC $t$-matrix to note at this point is its asymptotic behavior at large energies, as has already been assumed in Eq. (3.17). This behavior was used in Section 3 as the definition of an SHC potential.

\section{The Half-Shell BG Transition Amplitude for the Pure HC Interaction}

In the case of the singular pure $\mathrm{HC}$ interaction, the ordinary BG equation (2.2) for the transition amplitude or $T$-matrix in the many-body background is illdefined. Instead (in the case of zero total momentum, $\mathbf{P}=0$, of the interacting 
pair) we shall define the BG $T$-matrix in this case by the solution to Eq. (2.9). As a first step towards the general solution for the pure $\mathrm{HC}$ interaction, in this Section we investigate this integral equation for the case $s=p^{2}$, which gives as an equation for the half-shell $T$-matrix,

$$
{ }^{\circ} F_{l}\left(p, p^{\prime}\right)=\ddot{f}_{l}\left(p, p^{\prime}\right)+\int_{0}^{k_{F}} \frac{q^{2} d q}{2 \pi^{2}} \frac{t_{l}\left(p, q ; p^{\prime 2}+i \eta\right)}{q^{2}-p^{\prime 2}-i \eta} F_{l}\left(q, p^{\prime}\right) .
$$

Inserting the explicit forms for the free-scattering $t$-matrices from Eqs. (4.1), and exploiting its symmetry property, gives

$$
\begin{aligned}
F_{l}\left(p, p^{\prime}\right)= & \tilde{f}_{l}\left(p, p^{\prime}\right)+\int_{0}^{k_{F}} \frac{q^{2} d q}{2 \pi^{2}} \theta_{l}(p, q) F_{l}\left(q, p^{\prime}\right) \\
& +\tilde{f}_{l}\left(p, p^{\prime}\right) \int_{0}^{k_{F}} \frac{q^{2} d q}{2 \pi^{2}} \frac{b_{l}\left(q, p^{\prime}\right)}{q^{2}-p^{\prime 2}-i \eta} F_{l}\left(q, p^{\prime}\right) .
\end{aligned}
$$

This equation is then slightly rewritten as

$$
F_{l}\left(p, p^{\prime}\right)=d_{l}(p) M_{l}\left(p^{\prime}\right)+\left(2 \pi^{2}\right)^{-1} \int_{0}^{k_{F}} q^{2} d q \theta_{l}(p, q) F_{l}\left(q, p^{\prime}\right)
$$

where

$$
M_{l}\left(p^{\prime}\right)=m_{l}\left(p^{\prime}\right)\left[1+\int_{0}^{k_{F}}\left(q^{2} d q / 2 \pi^{2}\right)\left(b_{l}\left(q, p^{\prime}\right) /\left(q^{2}-p^{\prime 2}-i \eta\right)\right) F_{l}\left(q, p^{\prime}\right)\right],
$$

in terms of the notation introduced in Eqs. (4.9) and (4.10).

Treating $M_{l}\left(p^{\prime}\right)$ as a known function, it is clear by iteration of Eq. (5.3) that there is a separable solution for the amplitude $F_{l}\left(p, p^{\prime}\right)$,

$$
F_{l}\left(p, p^{\prime}\right)=D_{l}(p) M_{l}\left(p^{\prime}\right),
$$

where $D_{l}(p)$ is a real function which satisfies the integral equation,

$$
D_{l}(p)=d_{l}(p)+\left(2 \pi^{2}\right)^{-1} \int_{0}^{k_{F}} q^{2} d q \theta_{l}(p, q) D_{l}(q) .
$$

The function $M_{l}(p)$ is in turn given explicitly in terms of the solution $D_{l}(p)$ to this equation, by

$$
\frac{m_{l}(p)}{M_{l}(p)}=1-m_{l}(p) \int_{0}^{k_{F}} \frac{q^{2} d q}{2 \pi^{2}} \frac{b_{l}(q, p) D_{l}(q)}{q^{2}-p^{2}-i \eta} .
$$

The solution for the half-shell BG transition amplitude is thus wholly given in terms of known functions, once Eq. (5.6) has been solved for the unknown function 
$D_{l}(p)$. Equation (5.6) is a Fredholm integral equation, and by the well-known theory [13] of such integral equations it can be asserted that the solution is unique so long as the homogeneous equations has no nontrivial solution; that is so long as the equation

$$
\phi_{l}(p)=\lambda\left(2 \pi^{2}\right)^{-1} \int_{0}^{k_{F}} q^{2} d q \theta_{l}(p, q) \phi_{l}(q),
$$

has no eigenvalue $\lambda$ equal to unity. This equation is studied in Appendix A, where it is shown that for all partial waves all eigenvalues are greater than unity, for all finite values of both the Fermi momentum $k_{F}$ and the $\mathrm{HC}$ range $a$. We can therefore assert that the half-shell $\mathrm{BG} T$-matrix for the pure $\mathrm{HC}$ interaction has a unique solution, given by Eqs. (5.5)-(5.7).

In terms of the integrals ${ }^{2} I_{1, l}^{<}(p)$ and $I_{2, l}^{<}(p)$, defined as

$$
\begin{aligned}
& I_{1, l}^{<}(p) \equiv \int_{0}^{k_{F}} \frac{q^{2} d q}{2 \pi^{2}} \frac{d_{l}(q) D_{l}(q)}{q^{2}-p^{2}-i \eta}, \\
& I_{2, l}^{<}(p) \equiv \int_{0}^{k_{F}} \frac{q^{2} d q}{2 \pi^{2}} \frac{c_{l}(q) D_{l}(q)}{q^{2}-p^{2}-i \eta},
\end{aligned}
$$

the defining integral equation (5.6) for the function $D_{l}(p)$ can be written as

$$
D_{l}(p)=d_{l}(p)+c_{l}(p) I_{1, l}^{<}(p)-d_{l}(p) I_{2, l}^{<}(p),
$$

where use has been made of Eq. (4.10). Similarly using Eq. (4.10), the expression (5.7) for $M_{l}(p)$ can be written as

$$
\frac{m_{l}(p)}{M_{l}(p)}=1-I_{2, l}^{<}(p)-\frac{m_{l}(p)-c_{l}(p)}{d_{l}(p)} I_{1, l}^{<}(p) .
$$

These last two equations can be solved for $I_{1, l}^{<}$and $I_{2, l}^{<}$to give

$$
\begin{aligned}
& I_{1, l}^{<}(p)=\frac{D_{l}(p)}{m_{l}(p)}-\frac{d_{l}(p)}{M_{l}(p)}, \\
& I_{2, l}^{<}(p)=1-\frac{c_{l}(p)}{M_{l}(p)}-\frac{D_{l}(p)}{d_{l}(p)}\left[1-\frac{c_{l}(p)}{m_{l}(p)}\right] .
\end{aligned}
$$

The first of these last results confirms the identity obtained from the fully on-shell version of Eq. (5.1), namely, that obtained by putting $p=p^{\prime}$. Since $d_{l}(p)$ is a

${ }^{2}$ The superscripts $<$ and $>$ are employed consistently when defining integrals $I_{n, 2}$ to indicate that the range of integration is $\left(0, k_{F}\right)$ or $\left(k_{F}, \infty\right)$, respectively. The absence of a superscript indicates that the integral is defined over the full range $(0, \infty)$. Thus, $I_{n, l}=I_{n, l}^{<}+I_{n, l}^{>}$. 
real function by definition, and hence from Eq. (5.6) so is $D_{l}(p)$, Eq. (5.12) gives trivially that

$$
\operatorname{Im} I_{1, l}^{<}(p)=D_{l}(p)(4 \pi)^{-1} p d_{l}(p)-d_{l}(p) \operatorname{Im}\left[1 / M_{l}(p)\right],
$$

where we have used the explicit definitions (4.9). Comparison with the definition of $I_{1, l}^{<}(p)$ from Eq. (5.9) immediately yields the important result,

$$
\operatorname{Im}\left[1 / M_{l}(p)\right]=(4 \pi)^{-1} p D_{l}(p) \theta\left(p-k_{F}\right) .
$$

Having found the solution for the half-shell BG $T$-matrix for the pure HC interaction in terms of the solution $D_{l}(p)$ to a simple one-dimensional Fredholm integral equation, we shall show in Section 6 that the fully off-shell BG $T$-matrix for this interaction also can be solved in terms of just this solution. Before proceeding to the fully off-sheil $T$-matrix however, it is instructive to examine how the half-shell solution already obtained leads to a BG wavefunction which vanishes identically inside the hard core.

From Eq. (2.14a), the BG wavefunction for zero total momentum of the interacting pair in the $l$ th partial wave, is given by

$$
\Psi_{p, l}(r)=j_{l}(p r)-\int_{k_{F}}^{\infty} \frac{q^{2} d q}{2 \pi^{2}} \frac{j_{l}(q r) F_{l}(q, p)}{q^{2}-p^{2}-i \eta} .
$$

Using the results of Eqs. (5.5) and (5.13), this expression can be rewritten in the form

$$
\Psi_{p, l}(r)=j_{l}(p r)-M_{l}(p) \frac{2}{\pi} \int_{0}^{\infty} q d q \frac{j_{l}(q r) \operatorname{Im}\left[1 / M_{l}(q)\right]}{q^{2}-p^{2}-i \eta} .
$$

The remaining integral in Eq. (5.14) can be evaluated by making use of the analytic properties of the function $M_{l}(p)$. From Eq. (5.11) we have the result,

$$
1 / M_{l}(p)=i p a^{2} h_{l}^{+}(p a)\left[1-I_{2, l}^{<}(p)\right]+(4 \pi)^{-1} i p^{2} h_{l}^{+\prime}(p a) I_{1, l}^{<}(p),
$$

which immediately implies that the function $M_{l}^{-1}(p)$ is analytic in the entire complex $p^{2}$-plane, with the exception of a pole of order $l$ at the origin, and a cut on the right-hand real axis. Using these properties, and the fact that the Bessel function $j_{l}(z)$ is an entire function of the complex variable $z$, with a zero of order $l$ at the origin, it is clear that the product $j_{l}(p r) M_{l}^{-1}(p)$ is analytic in the entire cut $p^{2}$-plane. The integral is thus easily evaluated by considering the contour integral

$$
\frac{1}{2 \pi i} \oint_{C} d q^{2} \frac{j_{l}(q r) / M_{l}(q)}{q^{2}-p^{2}-i \eta}
$$


where $C$ is the contour shown in Fig. 1. The asymptotic properties for large $z$ of the spherical Bessel functions and of the function $M_{l}^{-1}\left(z^{1 / 2}\right)$ obtained explicitly from Eq. (5.15), show that the contribution from the infinite circular arc to the contour integral vanishes if and only if $r \leqslant a$. Comparison of the results evaluating the contour integral directly and by use of the Cauchy residue theorem thus gives

$$
\frac{1}{\pi} \int_{0}^{\infty} d q^{2} \frac{j_{l}(q r) \operatorname{Im}\left[1 / M_{l}(q)\right]}{q^{2}-p^{2}-i \eta}=j_{l}(p r) / M_{l}(p) ; \quad r \leqslant a .
$$

Comparison with Eq. (5.14) then shows that the BG wavefunction $\Psi_{p, l}(r)$ vanishes identically for $r \leqslant a$, as required.

\section{The Fully Off-Shell BG $T$-matrix for the Pure HC Interaction}

In the case of the pure $\mathrm{HC}$ interaction, the defining integral equation (2.9) for the BG $T$-matrix is readily written in the following form by making use of Eqs. (4.1) and (4.10),

$$
\begin{aligned}
T_{l}\left(p, p^{\prime} ; s+i \eta\right)= & \left(p^{\prime 2}-s\right) \theta_{l}\left(p, p^{\prime}\right)+d_{l}(p) M_{l}(\kappa) B_{l}\left(p^{\prime}, \kappa\right) \\
& +\left(2 \pi^{2}\right)^{-1} \int_{0}^{k_{F}} q^{2} d q \theta_{l}(p, q) T_{l}\left(q, p^{\prime} ; s+i \eta\right),
\end{aligned}
$$

where

$$
B_{l}\left(p^{\prime}, \kappa\right)=\frac{m_{l}(\kappa)}{M_{l}(\kappa)}\left[b_{l}\left(p^{\prime}, \kappa\right)+\int_{0}^{k_{F}} \frac{q^{2} d q}{2 \pi^{2}} \frac{b_{l}(q, \kappa) T_{l}\left(q, p^{\prime} ; s+i \eta\right)}{q^{2}-s-i \eta}\right] .
$$

Treating $B_{l}\left(p^{\prime}, \kappa\right)$ as a known function enables us to use the results of Appendix A again in asserting that the above integral equation is of the Fredholm type, with a unique solution. In particular, the equation can be iterated to yield the solution, with the result

$$
T_{l}\left(p, p^{\prime} ; s+i \eta\right)=\left(p^{\prime 2}-s\right) \Theta_{l}\left(p, p^{\prime}\right)+F_{l}(p, \kappa) B_{l}\left(p^{\prime}, \kappa\right),
$$

where the function $\Theta_{l}$ satisfies the integral equation (3.19S), and where the defining equation (5.6) for the function $D_{l}(p)$ has been used. It is clear by iteration of Eq. (3.19S) that $\Theta_{l}\left(p, p^{\prime}\right)$ is a symmetric function,

$$
\Theta_{l}\left(p, p^{\prime}\right)=\Theta_{l}\left(p^{\prime}, p\right),
$$

since the function $\theta_{l}\left(p, p^{\prime}\right)$ is also symmetric. Making use of the fact that $T_{l}\left(p, p^{\prime}\right.$; $s+i \eta)$ is symmetric under the interchange of $p$ and $p^{\prime}$ from Eq. (3.5), and using 
the explicit form of the solution given in Eq. (6.2), implies that $\Theta_{l}\left(p, p^{\prime}\right)$ satisfies the relation

$$
\Theta_{l}\left(p, p^{\prime}\right)=\frac{D_{l}\left(p^{\prime}\right) M_{l}(\kappa) B_{l}(p, \kappa)-D_{l}(p) M_{l}(\kappa) B_{l}\left(p^{\prime}, \kappa\right)}{p^{\prime 2}-p^{2}} .
$$

Since the function $\Theta_{l}\left(p, p^{\prime}\right)$ is independent of the parameter $\kappa$ according to the solution (6.2), this last functional relation implies that $B_{l}(p, \kappa)$ has the form

$$
M_{l}(\kappa) B_{l}(p, \kappa)=D_{l}(p) A_{l}(\kappa)+C_{l}(p),
$$

where $C_{l}(p)$ and $A_{l}(p)$ are arbitrary functions. However, from the definition of Eq. (6.1) directly, together with Eq. (5.7), we have

$$
B_{l}(\kappa, \kappa)=1,
$$

which is also necessary to satisfy Eq. (6.2) when $p^{\prime}=\kappa$. Putting $p=\kappa$ in Eq. (6.4) thus enables us to eliminate the unknown function $A_{l}(p)$ to get the result

$$
M_{l}(\kappa) B_{l}(p, \kappa)=C_{l}(p)+D_{l}(p)\left[M_{l}(\kappa)-C_{l}(\kappa)\right] / D_{l}(\kappa),
$$

which in its turn implies

$$
\Theta_{l}\left(p, p^{\prime}\right)=\frac{D_{l}\left(p^{\prime}\right) C_{l}(p)-D_{l}(p) C_{l}\left(p^{\prime}\right)}{p^{\prime 2}-p^{2}} .
$$

Since the function $C_{l}(p)$ is the one remaining undetermined function in the solution for the fully off-shell $\mathrm{BG} T$-matrix, we shall accordingly investigate its properties further. Substituting the above form for $\Theta_{l}\left(p, p^{\prime}\right)$ into its defining integral equation (3.19S), we have

$$
\begin{aligned}
& \Theta_{l}\left(p, p^{\prime}\right)-\theta_{l}\left(p, p^{\prime}\right) \\
& \quad=C_{l}\left(p^{\prime}\right) \int_{0}^{k_{F}} \frac{q^{2} d q}{2 \pi^{2}} \frac{\theta_{l}(p, q) D_{l}(q)}{q^{2}-p^{\prime 2}-i \eta}-D_{l}\left(p^{\prime}\right) \int_{0}^{k_{F}} \frac{q^{2} d q}{2 \pi^{2}} \frac{\theta_{l}(p, q) C_{l}(q)}{q^{2}-p^{\prime 2}-i \eta},
\end{aligned}
$$

where the factors $i \eta$ in the denominators have been inserted for convenience. The first integral can now readily be evaluated upon inserting from Eq. (4.10) for $\theta_{l}(p, q)$;

$$
\begin{aligned}
I_{3, l}^{<}\left(p, p^{\prime}\right) \equiv & \int_{0}^{k_{F}} \frac{q^{2} d q}{2 \pi^{2}} \frac{\theta_{l}(p, q) D_{l}(q)}{q^{2}-p^{2}-i \eta} \\
= & \frac{1}{p^{2}-p^{2}} \int_{0}^{k_{F}} \frac{q^{2} d q}{2 \pi^{2}}\left[d_{l}(q) c_{l}(p)-c_{l}(q) d_{l}(p)\right] \\
& \times D_{l}(q)\left[\frac{1}{q^{2}-p^{2}-i \eta}-\frac{1}{q^{2}-p^{\prime 2}-i \eta}\right] .
\end{aligned}
$$


Use of Eqs. (5.9) and (5.12), together with the expression (4.10) for the function $b_{l}\left(p, p^{\prime}\right)$, readily yields the result

$$
I_{3, l}^{<}\left(p, p^{\prime}\right)=\frac{1}{p^{2}-p^{\prime 2}}\left[D_{l}(p)-b_{l}\left(p, p^{\prime}\right) D_{l}\left(p^{\prime}\right)\right]-\frac{\theta_{l}\left(p, p^{\prime}\right)}{M_{l}\left(p^{\prime}\right)} .
$$

Using this result in Eq. (6.8) gives,

$$
\begin{aligned}
I_{6, l}^{<}\left(p, p^{\prime}\right) & =\int_{0}^{k_{F}} \frac{q^{2} d q}{2 \pi^{2}} \frac{\theta_{l}(p, q) C_{l}(q)}{q^{2}-p^{\prime 2}-i \eta} \\
& =\left(p^{2}-p^{\prime 2}\right)^{-1}\left[C_{l}(p)-b_{l}\left(p, p^{\prime}\right) C_{l}\left(p^{\prime}\right)\right]-Z_{l}\left(p^{\prime}\right) \theta_{l}\left(p, p^{\prime}\right)
\end{aligned}
$$

where

$$
Z_{l}(p)=\frac{1}{D_{l}(p)}\left[\frac{C_{l}(p)}{M_{l}(p)}-1\right] .
$$

Inserting the expression (4.10) for $\theta_{l}\left(p, p^{\prime}\right)$ in Eq. (6.11) gives the result

$$
\begin{gathered}
\frac{b_{l}\left(p, p^{\prime}\right)}{d_{l}(p)} C_{l}\left(p^{\prime}\right)-\frac{C_{l}(p)}{d_{l}(p)}+Z_{l}\left(p^{\prime}\right)\left[c_{l}\left(p^{\prime}\right)-c_{l}(p) \frac{d_{l}\left(p^{\prime}\right)}{d_{l}(p)}\right] \\
=\frac{c_{l}(p)}{d_{l}(p)}\left[I_{4, l}^{<}\left(p^{\prime}\right)-I_{4, l}^{<}(p)\right]+I_{5, l}^{<}(p)-I_{5, l}^{<}\left(p^{\prime}\right),
\end{gathered}
$$

where

$$
\begin{aligned}
& I_{4, l}^{<}(p) \equiv \int_{0}^{k_{F}} \frac{q^{2} d q}{2 \pi^{2}} \frac{d_{l}(q) C_{l}(q)}{q^{2}-p^{2}-i \eta} ; \\
& I_{5, l}^{<}(p) \equiv \int_{0}^{k_{F}} \frac{q^{2} d q}{2 \pi^{2}} \frac{c_{l}(q) C_{l}(q)}{q^{2}-p^{2}-i \eta} .
\end{aligned}
$$

By adding to Eq. (6.13) the corresponding expression obtained by the interchange of $p$ and $p^{\prime}$, it is easy to obtain the functional relation,

$$
\begin{aligned}
I_{4, l}^{<}(p) & +Z_{l}(p) d_{l}(p)-\left(C_{l}(p) / m_{l}(p)\right) \\
= & I_{4, l}^{<}\left(p^{\prime}\right)+Z_{l}\left(p^{\prime}\right) d_{l}\left(p^{\prime}\right)-\left(C_{l}\left(p^{\prime}\right) / m_{l}\left(p^{\prime}\right)\right),
\end{aligned}
$$

which evidently has the solution

$$
I_{4, l}^{<}(p)=\left(C_{l}(p) / m_{l}(p)\right)-Z_{l}(p) d_{l}(p)-k_{1},
$$

where $k_{1}$ is an arbitrary constant, independent of $p$. Inserting this result in the expression (6.13) gives the corresponding equation,

$$
I_{5, l}^{<}(p)=\left(1 / d_{l}(p)\right)\left[\left(c_{l}(p) / m_{l}(p)\right)-1\right] C_{l}(p)-Z_{l}(p) c_{l}(p)-k_{2},
$$


where $k_{2}$ is another arbitrary constant. The last two equations represent a pair of singular integral equations of the Omnès-Muskhelishvili type [14], neither of which has a unique solution, but which taken together presumably suffice to uniquely determine the function $C_{l}(p)$ and also the constants $k_{1}$ and $k_{2}$. Rather than trying to solve these equations directly however, they can be simply transformed into a nonsingular Fredholm equation, which will thereby provide us with more insight into the solution. Accordingly, Eqs. (6.15) and (6.16) are multiplied respectively by $c_{l}(p)$ and $d_{l}(p)$, and upon taking the resulting difference we obtain,

$$
C_{l}(p)=k_{1} c_{l}(p)-k_{2} d_{l}(p)+\left(2 \pi^{2}\right)^{-1} \int_{0}^{k_{F}} q^{2} d q \theta_{l}(p, q) C_{l}(q) .
$$

The remarkable similarity between Eq. (6.17) and the defining integral equation (5.6) for $D_{l}(p)$ should be noted. We can again immediately assert that the solution $C_{l}(p)$ to Eq. (6.18) for given values of the constants $k_{1}$ and $k_{2}$ is unique by the same arguments used for $D_{l}(p)$ above, utilizing the results of Appendix A. We note in passing that $k_{1}$ and $k_{2}$ can still depend on the only dimensionless parameter pertaining to the problem, namely $c \equiv k_{\mathrm{F}} a$.

In order to obtain the solution for $C_{l}(p)$, we shall henceforth focus attention on Eq. (6.17), and as a preliminary step in evaluating the constant $k_{1}$, we consider the integral,

$$
I_{7, l}^{<}(p) \equiv \int_{0}^{k_{F}} \frac{k^{2} d k}{2 \pi^{2}} \frac{D_{l}(k) C_{l}(k)}{k^{2}-p^{2}-i \eta}
$$

After Eq. (6.17) has been used to substitute for $C_{l}(k)$ in the integrand, the first two terms can be written in terms of the integrals $I_{1, l}^{<}$and $I_{2, l}^{<}$defined in Eq. (5.9). The third term which contains a double integration is evaluated by interchanging the order of the integrations and employing the results of Eqs. (6.9) and (6.10). Thus we obtain,

$$
\begin{aligned}
I_{7, l}^{<}(p)= & k_{1} I_{2, l}^{<}(\rho)-k_{2} I_{1, l}^{<}(p)+\int_{0}^{k_{F}} \frac{q^{2} d q}{2 \pi^{2}} C_{l}(q) \\
& \times\left\{\frac{1}{q^{2}-p^{2}-i \eta}\left[D_{l}(q)-b_{l}(q, p) D_{l}(p)\right]-\frac{\theta_{l}(q, p)}{M_{l}(p)}\right\}
\end{aligned}
$$

where we are at liberty to subtract the term i $\eta$ from the denominator of the first term in the integrand, since the numerator vanishes identically when $q=p$. The first term in the remaining integrand just cancels with $I_{7 . l}^{<}(p)$ on the left side of the equation. The second term in the integrand is rewritten using Eqs. (4.10) 
and (6.14); and the third term can be rewritten from the equation (6.17) being used to define $C_{l}(p)$. The following result is thereby obtained,

$$
\begin{aligned}
0= & k_{1} I_{2, l}^{<}(p)-k_{2} I_{1, l}^{<}(p)-\frac{D_{l}(p)}{m_{l}(p)}\left[I_{5, l}^{<}(p)+\frac{m_{l}(p)-c_{l}(p)}{d_{l}(p)} I_{4, l}^{<}(p)\right] \\
& -\frac{1}{M_{l}(p)}\left[C_{l}(p)-k_{1} c_{l}(p)+k_{2} d_{l}(p)\right] .
\end{aligned}
$$

Substituting the known expressions (5.12) for the integrals $I_{1, l}^{<}$and $I_{2, l}^{<}$and using the trivial identity,

$$
C_{l}(p)=c_{l}(p)\left[k_{1}+I_{4, l}^{<}(p)\right]-d_{l}(p)\left[k_{2}+I_{5, l}^{<}(p)\right],
$$

which follows from an obvious rewriting of Eq. (6.17), in order to eliminate the function $I_{5, l}^{<}$, it is then an easy matter to reduce Eq. (6.19) to the expression

$$
I_{4, l}^{<}(p)=\frac{C_{l}(p)}{m_{l}(p)}-\frac{d_{l}(p)}{D_{l}(p)}\left[\frac{C_{l}(p)}{M_{l}(p)}-k_{1}\right]-k_{1} .
$$

Direct comparison of this result with Eqs. (6.15) and (6.12) leads immediately to the conclusion

$$
k_{1}=1 \text {. }
$$

The only remaining arbitrariness in our solution for the fully off-shell BG $T$-matrix for the pure $\mathrm{HC}$ interaction at this point is the constant $k_{2}$. At first sight it appears unfortunate that $k_{2}$ has been eliminated from the expression (6.20) for $I_{4, l}^{<}(p)$ and one's first thoughts are to try a similar procedure as above but starting with a different integral expression. However, there is no means available to evaluate $k_{2}$ in this manner; it is in fact truly arbitrary. The point is that the solution given in Eq. (6.2) is invariant under the transformation,

$$
C_{l}(p) \rightarrow \tilde{C}_{l}(p)=C_{l}(p)+k D_{l}(p),
$$

where $k$ is an arbitrary constant, since from the explicit expressions of Eqs. (6.7) and (6.6) it is readily verified that both the functions $\Theta_{l}\left(p, p^{\prime}\right)$ and $B_{l}\left(p, p^{\prime}\right)$ remain invariant under this transformation. We can use this invariance to our advantage in the following manner. Making the transformation (6.22) in Eq. (6.17), and using the result $k_{1}=1$ and the defining Eq. (5.6) for $D_{l}(p)$ gives the integral equation satisfied by $\tilde{C}_{l}(p)$ as,

$$
\tilde{C}_{l}(p)=\left(k-k_{2}\right) d_{l}(p)+c_{l}(p)+\left(2 \pi^{2}\right)^{1} \int_{0}^{k_{F}} q^{2} d q \theta_{l}(p, q) \tilde{C}_{l}(q),
$$


where in particular we may choose $k=k_{2}$ if we wish. On the other hand by partially differentiating Eq. (5.6) with respect to the parameter $a$, and using the results,

$$
\begin{gathered}
c_{l}(p)=(\partial / \partial a)\left[\left(4 \pi a^{2}\right)^{-1} d_{l}(p)\right], \\
(\partial / \partial a) \theta_{l}\left(p, p^{\prime}\right)=\left(4 \pi a^{2}\right)^{-1} d_{l}(p) d_{l}\left(p^{\prime}\right),
\end{gathered}
$$

which follow immediately from Eqs. (4.5) and (4.9), we obtain the result,

$$
\begin{gathered}
(\partial / \partial a)\left[\left(4 \pi a^{2}\right)^{-1} D_{l}(p)\right] \\
=k_{3} d_{l}(p)+c_{l}(p)+\left(2 \pi^{2}\right)^{-1} \int_{0}^{k_{F}} q^{2} d q \theta_{l}(p, q)(\partial / \partial u)\left[\left(4 \pi a^{2}\right)^{-1} D_{l}(q)\right] \\
k_{3} \equiv\left(2 \pi^{2}\right)^{-1}\left(4 \pi a^{2}\right)^{-2} \int_{0}^{k_{F}} q^{2} d q d_{l}(q) D_{l}(q)
\end{gathered}
$$

By exactly the same arguments as before, it can be asserted that both Eqs. (6.23) and (6.25) have unique solutions. Thus, by comparing these equations with Eq. (6.17), and choosing $k_{2}=-k_{3}$, it is clear that the most general solution for the function $C_{l}(p)$ is

$$
C_{l}(p)=(\partial / \partial a)\left[\left(4 \pi a^{2}\right)^{-1} D_{l}(p)\right]+k D_{l}(p)
$$

where $k$ is an arbitrary constant that drops out from the expression for $T_{l}\left(p, p^{\prime}\right.$; $s+i \eta$ ), which is a result of surprising simplicity.

At this point we have arrived at a complete expression for the fully off-shell BG $T$-matrix for the case of zero total momentum of the pair interacting in the many-body medium. Using the results obtained it is now possible to verify that our solution obeys the various general relations obtained in Section 3. Thus the generalized unitarity relation of Eq. (3.8) is trivially proven using the result of Eq. (5.13). Similarly the generalized orthonormality relation of Eq. (3.9) can be verified by employing the integral expressions (B.12) and (B.13) evaluated in Appendix B; and the generalized completeness relations (3.21S) and (3.25) are readily proven using Eq. (B.14).

Before leaving the $\mathrm{HC}$ solution, it is instructive to derive one final relation for the function $M_{l}(p)$. Upon multiplying the integral $I_{1, l}^{<}(p)$ from Eq. (5.9) by the quantity $\left(4 \pi a^{2}\right)^{-2}$, and employing the result (5.12), it is easy to obtain upon partial differentiation with respect to the hard-core range parameter $a$, the relation

$$
\begin{aligned}
I_{2, l}^{<}(p)+I_{4, l}^{<}(p)= & \frac{C_{l}(p)}{m_{l}(p)}+D_{l}(p) \frac{\partial}{\partial a}\left[\frac{1}{4 \pi a^{2} m_{l}(p)}\right] \\
& -\frac{c_{l}(p)}{M_{l}(p)}-d_{l}(p) \frac{\partial}{\partial a}\left[\frac{1}{4 \pi a^{2} M_{l}(p)}\right]
\end{aligned}
$$


where the integrals $I_{2, l}^{<}$and $I_{4, l}^{<}$have already been defined in Eqs. (5.9) and (6.14), and where the results (6.24) and (6.26) have been employed, and the choice $k=0$ has been made purely for ease. Inserting the expression,

$$
\frac{\partial}{\partial a}\left[\frac{1}{4 \pi a^{2} m_{l}(p)}\right]=\frac{1}{d_{l}(p)}\left[\frac{c_{l}(p)}{m_{l}(p)}-1\right],
$$

which is easily derived from the explicit formulae (4.9), and using the results of Eqs. (5.12) and (6.15), it is readily shown that

$$
\frac{\partial}{\partial a}\left[\frac{1}{4 \pi a^{2} M_{l}(p)}\right]=\frac{1}{D_{l}(p)}\left[\frac{C_{l}(p)}{M_{l}(p)}-1\right] .
$$

The two results (6.27) and (6.28) are again remarkable for their similarity. Using Eq. (6.26) with $k=0$ once more, allows us to rewrite this last equation as,

$$
\frac{\partial}{\partial a}\left[\frac{1}{a^{2} M_{l}(p)}\right]=\frac{1}{a^{2} M_{l}(p)} \frac{\partial / \partial a\left[a^{-2} D_{l}(p)\right]}{a^{-2} D_{l}(p)}-\frac{4 \pi}{D_{l}(p)} .
$$

This equation can in turn be integrated to yield,

$$
\frac{1}{M_{l}(p ; a)}=D_{l}(p ; a)\left[\frac{1}{M_{l}\left(p ; a_{0}\right) D_{l}\left(p ; a_{0}\right)}-\int_{a_{0}}^{a} \frac{4 \pi a^{\prime 2} d a^{\prime}}{D_{l}^{2}\left(p ; a^{\prime}\right)}\right],
$$

where the dependence of the functions $D_{l}(p) \equiv D_{l}(p ; a)$ and $M_{l}(p) \equiv M_{l}(p ; a)$ on the hard core parameter $a$ has been written explicitly.

\section{EXTENSION TO THE BCM}

The results obtained in the last two sections for the pure $\mathrm{HC}$ interaction can now be easily generalized to the case of the pure boundary condition model (BCM). The most general boundary condition model for two particles consists of a core interaction, inside which the wavefunction vanishes, and which gives rise to an energy-independent logarithmic derivative of the wavefunction at the core radius $a$, beyond which a given local interaction operates. We shall consider only the pure boundary condition model (BCM) where the external potential is identically zero. The boundary condition on the two-particle wavefunction is taken to be

$$
\lim _{\delta \rightarrow 0}\left(\psi_{k, l}^{\prime}(a+\delta) / \psi_{k, l}(a+\delta)\right)=\lambda_{l}
$$


The BCM can be equivalently cast as the limiting form of a local interaction model containing a repulsive square well and an attractive surface delta-function potential [15],

$$
u(r)=g \theta(a-r)-h \delta(r-a)
$$

where $g>0, h>0$, and the limit as $g \rightarrow \infty, h \rightarrow \infty$ is taken such that the quantity

$$
\lambda=g^{1 / 2}-h-a^{-1},
$$

remains finite. The limit obviously can be taken separately in each partial wave. Thus the HC interaction is seen to be the special case of the BCM where $\lambda_{l} \rightarrow \infty$.

It can be shown [8] that the fully off-shell BCM $t$-matrix is expressed in terms of the half-shell quantity by exactly the same relation (4.1) as for the $\mathrm{HC}$ interaction, provided the attraction present is not strong enough to produce one or more bound states of a freely interacting pair. In all that follows we shall assume this to be the case. The only difference between the two expressions arises from the difference in the expressions for the half-shell $t$-matrices. For the BCM, the halfshell quantity $\tilde{f}_{l}(p, \kappa)$ is given by [8],

$$
\tilde{f}_{l}(p, \kappa)=\frac{4 \pi}{i \kappa} \frac{\lambda_{l} j_{l}(p a)-p j_{l}^{\prime}(p a)}{\lambda_{l} h_{l}^{+}(\kappa a)-\kappa h_{l}^{+{ }^{\prime}(\kappa a)}},
$$

which can be written in the nolation of Section 4 as

where

$$
\begin{aligned}
\tilde{f}_{l}(p, \kappa) & =\bar{d}_{l}(p) \bar{m}_{l}(\kappa), \\
d_{l}(p) & =d_{l}(p)-\mu_{l} c_{l}(p), \\
\bar{m}_{l}^{-1}(p) & =m_{l}^{-1}(p)-\mu_{i} z_{l}(p),
\end{aligned}
$$

$$
\begin{aligned}
z_{l}(p) & =d_{l}^{-1}(p)\left[c_{l}(p) m_{l}^{-1}(p)-1\right], \\
\mu_{l} & =4 \pi a^{2} / \lambda_{l} .
\end{aligned}
$$

Use has been made of the Wronskian relation (4.3) in writing $\bar{m}_{l}(p)$ in the form given in Eq. (7.2).

Repeating the calculations of Section 5, it is clear that the half-shell BG transition amplitude for the BCM is given by

$$
F_{l}(p, \kappa)=\bar{D}_{l}(p) \bar{M}_{l}(\kappa),
$$

where the function $\bar{D}_{l}(p)$ satisfies the integral equation

$$
\bar{D}_{i}(p)=\bar{d}_{l}(p)+\left(2 \pi^{2}\right)^{-1} \int_{0}^{k_{F}} q^{2} d q \theta_{l}(p, q) \bar{D}_{l}(q),
$$


and the function $\bar{M}_{l}(p)$ is given by

$$
\bar{M}_{l}^{-1}(p)=\bar{m}_{l}^{-1}(p)-\int_{0}^{k_{F}} \frac{q^{2} d q}{2 \pi^{2}} \frac{b_{l}(q, p) \bar{D}_{l}(q)}{q^{2}-p^{2}-i \eta} .
$$

Using Eqs. (5.6) and (6.17) with the result $k_{1}=1$ from Eq. (6.21) it is clear that $\bar{D}_{l}(p)$ is given by

$$
\bar{D}_{l}(p)=D_{l}(p)-\mu_{l} C_{l}(p),
$$

where $C_{l}(p)$ satisfies Eq. (6.17) with the constant $k_{2}$ set to zero. Using these results together with Eq. (6.25), it is seen that the function $C_{l}(p)$ in Eq. (7.7) may be written in the form,

$$
C_{l}(p)=(\partial / \partial a)\left[\left(4 \pi a^{2}\right)^{-1} D_{l}(p)\right]-k_{3} D_{l}(p),
$$

where $k_{3}$ is defined in Eq. (6.25).

Use of Eqs. (7.6) and (7.7), together with Eq. (5.7) enables us to write

$$
\bar{M}_{l}^{-1}(p)=\bar{m}_{l}^{-1}(p)+M_{l}^{-1}(p)-m_{l}^{-1}(p)+\mu_{l} \int_{0}^{k_{F}} \frac{q^{2} d q}{2 \pi^{2}} \frac{b_{l}(q, p) C_{l}(q)}{q^{2}-p^{2}-i \eta} .
$$

Using the explicit expression for $b_{l}(q, p)$ from Eq. (4.10), enables us to evaluate the integral expression above in terms of the functions defined in Eq. (6.14). Making use of Eq. (7.2), we can write

$$
\bar{M}_{l}^{-1}(p)=M_{l}^{-1}(p)-\mu_{l} z_{l}(p)+\mu_{l}\left[m_{l}^{-1}(p) I_{5, l}^{<}(p)-z_{l}(p) I_{4, l}^{<}(p)\right] .
$$

Finally, use of Eqs. (6.15) and (6.16), taking $k_{2}=0$ as required, gives the result,

$$
\widehat{M}_{l}^{-1}(p)=M_{l}^{-1}(p)-\mu_{l} Z_{l}(p) .
$$

The half-shell BG transition amplitude for the BCM is thus given completely in terms of known functions by Eqs. (7.4) and (7.7)-(7.9).

The fully off-shell $T$-matrix for the BCM also can be obtained exactly as in Section 6 for the HC interaction. By direct analogy with Eqs. (6.2) and (6.6) we find for the BCM,

$$
T_{l}\left(p, p^{\prime} ; s+i \eta\right)=\left(p^{\prime 2}-s\right) \Theta_{l}\left(p, p^{\prime}\right)+F_{l}(p, \kappa) \bar{B}_{l}\left(p^{\prime}, \kappa\right)
$$

where

$$
\bar{B}_{l}(p, \kappa) \bar{M}_{l}(\kappa)=C_{l}(p)+\bar{D}_{l}(p)\left[\bar{M}_{l}(\kappa)-C_{l}(\kappa)\right] / \bar{D}_{l}(\kappa),
$$

with $\Theta_{l}\left(p, p^{\prime}\right)$ exactly as before, and $F_{l}(p, \kappa)$ given by Eq. (7.4). Using the results 
of Eqs. (7.7) and (7.9), it is not difficult to show that Eq. (7.11) can be manipulated to give

$$
\bar{B}_{l}(p, \kappa)=B_{l}(p, \kappa) .
$$

The remarkable result thus holds true that the BG fully off-shell $T$-matrix for the $\mathrm{BCM}$ is given in terms of the half-shell amplitude $F_{l}(p, \kappa)$ by exactly the same relation (6.2) as for the $\mathrm{HC}$ interaction, in complete analogy with the free $t$-matrix expression (4.1), which also holds for both the pure $\mathrm{HC}$ and BCM.

\section{SUMMARY}

We have discussed the general properties of the fully off-shell $T$-matrix which is the solution to the Bethe-Goldstone equation, and which describes the interaction of two fermions outside the Fermi sea as mediated by the many-body background of identical particles, in which they are embedded. In particular, generalized orthonormality and completeness relations have been derived, and the analytic properties of the $T$-matrix have been discussed. Great care was taken to indicate the role played by the two-body interaction in determining the properties of the $T$-matrix, and in particular the differences that arise between well-behaved nonsingular (NS) potentials and singular interactions of the hard core (SHC) type were carefully delineated. General expressions were derived for both cases which enable one to calculate the fully off-shell $T$-matrix from a knowledge of the half-shell quantity alone (together with a knowledge of the bound states if these exist). In this respect, the main difference between singular and nonsingular potentials was shown to lie in the asymptotic behavior of their respective $T$ matrices, as the $\mathrm{CM}$ energy variable tends to infinity. In the language of dispersion theory, the dispersion integrals for SHC interactions need one more subtraction than those for NS potentials. It is hoped that the detailed derivation of these results will prove useful in the case of other singular interactions not covered by the present work.

The general formalism was illustrated by application to two examples of singular interactions of some practical interest, namely, the pure hard-core interaction and the pure boundary condition model. In order to simplify the subsequent calculations, the interacting pair within the many-body medium was chosen to have equal and opposite momenta, in order that the Bethe-Goldstone equation could be uncoupled in a partial-wave expansion. Apart from being the case of most physical interest, the extension to nonzero total momentum of the pair is straightforward, at least within the usual angle-averaging approximation employed in most nuclear matter and other allied calculations. For both model interactions considered, exact expressions were obtained for the fully off-shell $T$-matrices, and each was given in terms of the solution to the same simple one-dimensional 
Fredholm integral equation. The exact analytic properties of the solutions were discussed, and were exploited to both prove the uniqueness of the solutions, and to verify that they satisfied the various general relations derived. The solutions for both models exhibit a remarkable similarity with the respective free-scattering $t$-matrices for the same model interactions, that describe the behaviour of a pair of particles interacting in vacuo.

Although strictly singular potentials of the type discussed by us probably do not exist in nature, real potentials are often well approximated as the limiting case of nonsingular potentials. In the appropriate mathematical limit, all relevant scattering functions then depend on one parameter less, which is a considerable saving. Our general formalism enables us to exploit this saving from the outset, and avoids the necessity of explicitly taking the limit in each separate case. The formalism is suitable for more general interactions which contain a smooth nonsingular potential outside the core region of the pure singular models discussed. For these more general cases, the $T$-matrix solution obtained in this work for the purely singular piece of the interaction almost certainly can be employed profitably as a startingpoint in those calculations where the external nonsingular potential is weak enough to be able to be treated as a perturbation.

An expression for the two-body BG $T$-matrix for any pair interaction often can be used to advantage as the starting point for calculations on the properties of many-fermion systems. Previous calculations [12] of dilute many-fermion systems interacting as hard spheres, for example, have effectively used only the first few terms of a series expansion for the BG $T$-matrix or its equivalent, and consequent nonphysical divergences have arisen in expressions for such physical quantities as the ground-state energy. The expressions derived in this work should provide a better input for such calculations, and might possibly enable them to be extended beyond the low-density regime where the series expansions are valid.

An alternative starting-point for all such many-body calculations is the so-called Galitskii-Feynman (GF) $T$-matrix, which is closely related to the Bethe-Goldstone (BG) $T$-matrix discussed in the present work. The relationships between these two quantities and their relative merits are discussed in [12]. The GF $T$-matrix has the great advantage that it treats simultaneously the interaction of a scattering pair inside the many-body medium both when they are particles outside the Fermi sea and holes within the Fermi sea. In this way particles and holes are treated symmetrically, and many more terms of the original many-body perturbation series in powers of the two-body potential are summed by the GF $T$-matrix than by the corresponding BG quantity. The relative importance of these extra hole-hole correlation terms grows as the density of the system increases, and some calculations [16] exist which indicate the importance of keeping such terms. The present work concerning the BG $T$-matrix can in such cases be extended to the GF $T$-matrix, which it closely resembles. 


\section{APPENDIX A}

Several of the integral equations of interest in this work, namely, those in Eqs. (5.6), (6.1), (6.18), (6.24), (6.25), and (7.5) can be written in the general form, for the unknown function $\chi_{l}(p)$,

$$
\chi_{l}(p)=f_{l}(p)+\left(2 \pi^{2}\right)^{-1} \int_{0}^{k_{F}} q^{2} d q \theta_{l}(p, q) \chi_{l}(q),
$$

where $f_{l}(p)$ is assumed to be a known function, and the kernel $\theta_{l}\left(p, p^{\prime}\right)$ is given by Eq. (4.5). The purpose of this Appendix is to prove that Eq. (A.1) always has a unique solution for all finite values of the parameters $k_{F}$, the Fermi momentum, and $a$, the hard core range. The kernel of Eq. (A.1) is certainly smooth and squareintegrable, and hence by the theory of Fredholm integral equations [13] it is guaranteed that the Fredholm alternative applies. Namely, it can be asserted that either Eq. (A.1) has a unique solution, or the homogenous equation

$$
\phi_{l}(p)=\lambda_{l}\left(2 \pi^{2}\right)^{-1} \int_{0}^{k_{F}} q^{2} d q \theta_{l}(p, q) \phi_{l}(q),
$$

has an eigenvalue $\lambda_{l}=1$. Our proof of the uniqueness of the solution to Eq. (A.1) will thus take the form of disproving this latter possibility.

The transformation of Eq. (A.2) to dimensionless coordinates,

$$
x=a p / c ; \quad y=a q / c,
$$

is first made, where $c \equiv k_{F} a$. Making the replacement

$$
v_{l}(x)=x \phi_{l}(c x / a),
$$

and using the definition (4.5) for the kernel $\theta_{l}(p, q)$, enables Eq. (A.2) to be rewritten in the form,

$$
\begin{gathered}
v_{l}(x)=\lambda_{l} \int_{0}^{1} d y k_{l}(x, y ; c) v_{l}(y), \\
k_{l}(x, y ; c)=2 \pi^{-1} \int_{0}^{c} d u x u j_{l}(x u) y u j_{l}(y u) .
\end{gathered}
$$

The kernel $k_{l}$ in the above equation is obviously both symmetric and positive definite, since the quadratic form

$$
Q[\psi] \equiv \int_{0}^{1} d x \int_{0}^{1} d y \psi(x) k_{l}(x, y ; c) \psi(y) \geqslant 0,
$$


obeys the above inequality for arbitrary (real) functions $\psi(x)$. The theory of such kernels [13] guarantees that the spectrum of eigenvalues of the kernel, say

$$
\lambda_{l, i} ; \quad i=0,1,2, \ldots, \infty,
$$

can be ordered such that

$$
0 \leqslant \lambda_{l, 0} \leqslant \lambda_{l, 1} \leqslant \cdots,
$$

where all the eigenvalues are nonnegative. The remainder of our proof now rests on demonstrating that $\lambda_{l, 0}>1$.

The theory of positive-definite symmetric Fredholm kernels [13] allows us to write a variational principle to determine $\lambda_{l, 0}$ as

$$
\lambda_{t, 0}=\min \left(\|\psi\|^{2} / Q[\psi]\right),
$$

where

$$
\|\psi\|^{2}=\int_{0}^{1} d x \psi^{2}(x)
$$

If we suppose that $\psi_{l, 0}(x ; c)$ is the eigenfunction corresponding to the exact lowest eigenvalue $\lambda_{l, 0}(c)$, for a particular choice of the parameter $c$, then Eq. (A.5) can be used to study the dependence of $\lambda_{l, 0}$ on this parameter. Thus, for a small change $\Delta c$ in $c$, the quantities $Q\left[\psi_{l, 0}\right]$ and $\left\|\psi_{l, 0}\right\|^{2}$ change by amounts which can be determined from the definitions (A.4) and (A.6) to be,

$$
\begin{aligned}
\Delta Q\left[\psi_{l, 0}\right]= & 2 \lambda_{l, 0}^{-1} \int_{0}^{1} d x \psi_{l, 0}(x ; c) \Delta \psi_{l, 0}(x ; c) \\
& +2 \pi^{-1}\left[\int_{0}^{1} d x \psi_{l, 0}(x ; c) x c j_{l}(x c)\right]^{2} \Delta c+O(\Delta c)^{2}, \\
\Delta\left\|\psi_{l, 0}\right\|^{2}= & 2 \int_{0}^{1} d x \psi_{l, 0}(x ; c) \Delta \psi_{l, 0}(x ; c)+O(\Delta c)^{2},
\end{aligned}
$$

where the defining eigenvalue equation (A.3) has bcen used in the former case. From the variational principle (A.5), the corresponding first-order change in $\lambda_{l, 0}$ is

$$
\Delta \lambda_{l, 0}=\frac{\Delta\left\|\psi_{l, 0}\right\|^{2}-\lambda_{l, 0} \Delta Q\left[\psi_{l, 0}\right]}{Q\left[\psi_{l, 0}\right]}
$$

and using the above expressions it is readily shown that,

$$
d \lambda_{l, 0} / d c=-2 \pi^{-1} \lambda_{l, 0}\left[\int_{0}^{1} d x \psi_{l, 0}(x ; c) x c j_{l}(x c)\right]^{2} / Q\left[\psi_{l, 0}\right]<0 .
$$


Also, by using the completeness property for spherical Bessel functions, it is clear from Eq. (A.3) that in the limit $c \rightarrow \infty$,

$$
k_{l}(x, y ; \infty)=\delta(x-y),
$$

the Dirac delta function. Hence, we obtain the trivial result

$$
\lambda_{l}(\infty)=1
$$

which, together with Eq. (A.7), proves the inequality

$$
\lambda_{l, 0}(c)>1
$$

for all finite values of the parameter $c$. This in turn completes the proof of the uniqueness of the solution to Eq. (A.1).

\section{APPENDIX B}

In this Appendix we wish to investigate the analytic properties of the functions $C_{l}(p), D_{l}(p)$, and $\Theta_{l}\left(p, p^{\prime}\right)$ which are defined to be the solutions to the integral equations (6.17), (5.6), and (3.19S), respectively. We shall then use these analyticity results to evaluate certain integrals containing these functions. To this end we shall first consider the inhomogeneous terms and the kernel of the last two of these integral equations. Using the analytic expressions (4.5) and (4.9) and elementary properties of the spherical Bessel functions, it is clear that both $d_{l}(p)$ and $\theta_{l}\left(p, p^{\prime}\right)$ considered as functions of the complex variable $p$ belong to the class $\mathscr{L}_{l}\{a ; p\}$; where we define a function $f_{l}(z)$ to belong to the class $\mathscr{L}_{l}\{a ; z\}$ if all of the following conditions are satisfied:

(i) $f_{l}(z)$ is an integral (or entire) function of the complex variable $z=$ $x+i y$, i.e., the function $f_{l}(z)$ is analytic throughout the entire complex plane except for an isolated singularity at infinity;

(ii) $\lim _{x \rightarrow \infty} e^{-a x} f_{l}(z)=0$;

(iii) $\lim _{x \rightarrow-\infty} e^{a x} f_{l}(z)=0$;

(iv) $f_{l}(x)=(-1)^{\iota} f_{l}(-x)$;

(v) $\lim _{x \rightarrow 0} x^{\epsilon-l} f_{l}(x)=0$, for $\epsilon>0$.

It is now an easy matter to show that these same properties hold for $D_{l}(p)$. The last two conditions are immediately seen to be true by direct inspection of Eq. (5.6). Further, since the kernel of this equation is an entire function of the variable $p$ (for fixed $q$ ), the integral itself will also be entire, so long as the integral 
exists for all (noninfinite) complex values of $p$. Since we know that the equation has a unique solution for real values of $p$ from Appendix $\mathrm{A}$, the convergence of the integral is guaranteed in this case. Using the boundedness of the kernel it is an easy matter to show that the function $D_{l}(p)$ is entire and satisfies the bounds of conditions (ii) and (iii). In a completely analogous fashion, using Eq. (3.19S), one can show that $\Theta_{l}\left(p, p^{\prime}\right)$ also belongs to the class $\mathscr{L}_{l}\{a ; p\}$.

It is now possible to derive several integrals which will be of use to us, which are applicable to any function $f_{i}(p)$ belonging to the class $\mathscr{L}_{2}\{a ; p\}$ and which thus can be applied to both $D_{l}(p)$ and $\Theta_{l}\left(p, p^{\prime}\right)$ as special cases of most interest. Consider first the integral,

$$
J_{l}(p) \equiv \frac{2}{\pi} \int_{0}^{\infty} q^{2} d q \frac{j_{l}(q r) f_{l}(q)}{q^{2}-p^{2}-i \eta} ; \quad r \geqslant a .
$$

Using the property (iv) above to extend the range of integration, we write

$$
J_{l}(p)=\frac{1}{2 \pi} \int_{-\infty}^{\infty} q^{2} d q \frac{\left[h_{l}^{+}(q r)+h_{l}-(q r)\right]}{(q-p-i \eta)(q+p+i \eta)} f_{l}(q) .
$$

The functions $h_{l}^{ \pm}(x)$ are entire functions in the upper and lower half-planes, respectively, with the exception of a pole of order $(l+1)$ at the origin. It is clear from property $(\mathrm{v})$ however that the products $\left[q^{2} h_{l} \pm(q r) f_{l}(q)\right]$ are analytic everywhere including the origin. The two integrals in Eq. (B.2) thus can be converted into contour integrals by the addition, respectively, of the infinite semicircles in the upper and lower half-planes. The only singularities of the integrands inside the contours thus arise from the simple poles at $q= \pm(p+i \eta)$. Further, properties (ii) and (iii), together with the asymptotic condition

$$
h_{l}^{ \pm}(x) \underset{x \rightarrow \infty}{\longrightarrow} i^{\mp(l+1)} x^{-1} e^{ \pm i x},
$$

imply that the contribution to the contour integrals from the infinite semicircular arcs will vanish. The Cauchy residue theorem immediately gives the result,

$$
J_{l}(p)=\frac{1}{2} i p\left[h_{l}+(p r) f_{l}(p)+h_{l}^{-}(-p r) f_{l}(-p)\right] .
$$

Using the relation,

$$
h_{l}^{-}(-x)=(-1)^{l} h_{l}+(x),
$$

together with property (iv) again, gives our basic result,

$$
\frac{2}{\pi} \int_{0}^{\infty} q^{2} d q \frac{j_{l}(q r) f_{l}(q)}{q^{2}-p^{2}-i \eta}-i p h_{l}+(p r) f_{l}(p) ; \quad r \geqslant a,
$$


for any function $f_{l}(p)$ belonging to the class $\mathscr{L}_{l}\{a ; p\}$. For example, putting $r=a$ in the above equation gives the result

$$
I_{1, l}(p) \equiv \int_{0}^{\infty} \frac{q^{2} d q}{2 \pi^{2}} \frac{d_{l}(q) D_{l}(q)}{q^{2}-p^{2}-i \eta}=\frac{D_{l}(p)}{m_{l}(p)},
$$

using Eq. (4.9). Again, by putting $r=a$ and $f_{l}(p)=\Theta_{l}\left(p, p^{\prime}\right)$ in Eq. (B.3) gives

$$
\int_{0}^{\infty} \frac{q^{2} d q}{2 \pi^{2}} \frac{d_{l}(q) \Theta_{l}\left(q, p^{\prime}\right)}{q^{2}-p^{2}-i \eta}=\frac{\Theta_{l}\left(p, p^{\prime}\right)}{m_{l}(p)}
$$

By using the explicit representation (6.7) for $\Theta_{l}\left(p, p^{\prime}\right)$, Eqs. (B.4) and (B.5) together imply the relation,

$$
I_{4, l}(p) \equiv \int_{0}^{\infty} \frac{q^{2} d q}{2 \pi^{2}} \frac{d_{l}(q) C_{l}(q)}{q^{2}-p^{2}-i \eta} \doteq \frac{C_{l}(p)}{m_{l}(p)},
$$

where the symbol $\doteq$ indicates equality only as a once-subtracted relation; thus

$$
A(p) \doteq B(p) \Rightarrow A(p)-A\left(p^{\prime}\right)=B(p)-B\left(p^{\prime}\right) .
$$

We can also use Eq. (B.3) to evaluate the integral,

$$
I_{3, l}\left(p, p^{\prime}\right) \equiv \int_{0}^{\infty} \frac{q^{2} d q}{2 \pi^{2}} \frac{\theta_{l}(p, q) D_{l}(q)}{q^{2}-p^{\prime 2}-i \eta}
$$

Using the representation of Eq. (4.5) together with the completeness relation,

$$
\int_{0}^{\infty} r^{2} d r j_{l}(p r) j_{l}\left(p^{\prime} r\right)=\frac{1}{2} \pi p^{-2} \delta\left(p-p^{\prime}\right)
$$

the integral (B.7) can be rewritten as,

$$
\begin{aligned}
I_{3, l}\left(p, p^{\prime}\right) & =\int_{0}^{\infty} \frac{q^{2} d q}{2 \pi^{2}} \frac{D_{l}(q)}{q^{2}-p^{\prime 2}-i n}\left[\frac{2 \pi^{2}}{q^{2}} \delta(p-q)-4 \pi \int_{a}^{\infty} r^{2} d r j_{l}(p r) j_{l}(q r)\right] \\
& =\left(p^{2}-p^{\prime 2}\right)^{-1} D_{l}(p)-\int_{a}^{\infty} r^{2} d r j_{l}(p r) i p^{\prime} h_{l}{ }^{+}\left(p^{\prime} r\right) D_{l}\left(p^{\prime}\right),
\end{aligned}
$$

where the order of integration has been interchanged in the second term, and use has been made of Eq. (B.3). Using Eq. (4.2) enables us to write the final result,

$$
I_{3, l}\left(p, p^{\prime}\right)=\left[D_{l}(p)-b_{l}\left(p, p^{\prime}\right) D_{l}\left(p^{\prime}\right)\right] /\left(p^{2}-p^{\prime 2}\right) .
$$


Exactly as before, Eqs. (B.4) and (B.8) together imply,

$$
I_{2, l}(p)=\int_{0}^{\infty} \frac{q^{2} d q}{2 \pi^{2}} \frac{c_{l}(q) D_{l}(q)}{q^{2}-p^{2}-i \eta} \doteq \frac{D_{l}(p)}{d_{l}(p)}\left[\frac{c_{l}(p)}{m_{l}(p)}-1\right]
$$

and a more detailed calculation similar to that leading to Eq. (B.3) shows that Eq. (B.9) is an actual equality.

In order to prove that our solution for the BG $T$-matrix for the pure HC interaction satisfies the generalized orthogonality relations (3.9) and (3.10), it will suffice to evaluate the integrals,

$$
\begin{aligned}
& I_{7, l}^{>}(p) \equiv \int_{k_{F}}^{\infty} \frac{k^{2} d k}{2 \pi^{2}} \frac{D_{l}(k) C_{l}(k)}{k^{2}-p^{2}-i \eta}, \\
& I_{8 . l}^{>}(p) \equiv \int_{k_{F}}^{\infty} \frac{k^{2} d k}{2 \pi^{2}} \frac{D_{l}^{2}(k)}{k^{2}-p^{2}-i \eta} .
\end{aligned}
$$

The former of these integrals is evaluated by substituting for the function $C_{l}(k)$ from its defining integral equation (6.17),

$$
\begin{aligned}
I_{7, l}(p)= & \int_{k_{F}}^{\infty} \frac{k^{2} d k}{2 \pi^{2}} \frac{D_{l}(k)}{k^{2}-p^{2}-i \eta} \\
& \times\left[k_{1} c_{l}(k)-k_{2} d_{l}(k)+\left(2 \pi^{2}\right)^{-1} \int_{0}^{k_{F}} q^{2} d q \theta_{l}(k, q) C_{l}(q)\right]
\end{aligned}
$$

The first two terms in the above expression are readily evaluated using Eqs. (5.12), (B.4), and (B.9). The last term is evaluated by interchanging the order of integration and using the results of Eqs. (6.9) and (B.8) to enumerate the $k$-integration. We thus have the result,

$$
I_{7, l}^{>}(p)=\left[k_{1} c_{l}(p)-k_{2} d_{l}(p)+\left(2 \pi^{2}\right)^{-1} \int_{0}^{k_{F}} q^{2} d q \theta_{l}(q, p) C_{l}(q)\right] / M_{l}(p)-k_{1},
$$

which on reuse of Eq. (6.17) simplifies to the final expression,

$$
I_{7, l}^{>}(p)=C_{l}(p) / M_{l}(p)-1,
$$

where we have used the result $k_{1}=1$ from Eq. (6.21). The integral $l_{8 . l}^{>}(p)$ is evaluated in a completely analogous fashion, by use of its defining integral equation (5.6), to give the result

$$
I_{8 . l}^{>}(p)=D_{l}(p) / M_{l}(p)
$$


It is possible to analytically continue the definitions of Eqs. (B.10) and (B.11) to define functions $I_{7, l}^{>}\left(z^{1 / 2}\right)$ and $I_{8, l}^{>}\left(z^{1 / 2}\right)$ by simply making the replacement $p^{2}+i \eta \rightarrow z$ in the denominators of the integrands. From these definitions it is clear that both functions are analytic in the entire complex $z$-plane except for the cut on the right-hand real laxis. Furthermore, from the extended definition of Eq. (B.11) it is evident that the function $I_{8, l}^{>}\left(z^{1 / 2}\right)$ can have no zeros in the entire complex $z$-plane, with the exception of the cut along the real axis from $k_{F}^{2}$ to infinity. It can therefore be asserted that the function

$$
g\left(z^{1 / 2}\right) \equiv I_{7, l}^{>}\left(z^{1 / 2}\right) / I_{8, l}^{>}\left(z^{1 / 2}\right),
$$

is analytic in the entire complex $z$-plane, cut along the segment $\left(k_{F}^{2}, \infty\right)$ of the real axis. It is thus possible to write a subtracted dispersion relation for the function $g\left(z^{1 / 2}\right)$ by integrating around the contour $C$ shown in Fig. 1. The contribution from the infinite circular arc in the subtracted integral is readily seen to be zero, using the explicit asymptotic behavior of $g\left(z^{1 / 2}\right)$ for large $z$ obtained from the extended definitions of Eqs. (B.10) and (B.11). We can hence write,

$$
g\left(z^{1 / 2}\right) \doteq \frac{2}{\pi} \int_{k_{F}}^{\infty} q d q \frac{\operatorname{Im} g(q)}{q^{2}-z}
$$

Using Eqs. (B.12) and (B.13) enables us to write

$$
\operatorname{Im} g(p)=-\frac{1}{D_{l}(p)} \operatorname{Im} M_{l}(p)=\frac{\left|M_{l}(p)\right|^{2}}{D_{l}(p)} \operatorname{Im}\left[\frac{1}{M_{l}(p)}\right],
$$

which together with the explicit result of Eq. (5.13) gives the final result, on putting $z=p^{2}+i \eta$,

$$
\int_{k_{F}}^{\infty} \frac{q^{2} d q}{2 \pi^{2}} \frac{\left|M_{l}(q)\right|^{2}}{q^{2}-p^{2}-i \eta} \doteq \frac{C_{l}(p)-M_{l}(p)}{D_{l}(p)}
$$

\section{REFERENCES}

1. K. A. Brueckner and C. A. Levinson, Phys. Rev. 97 (1955), 1344; K. A. Brueckner, Phys. Rev. 100 (1955), 36.

2. J. Goldstone, Proc. Roy. Soc. (London) A239 (1957), 267.

3. B. A. Lippmann and J. Schwinger, Phys. Rev. 79 (1950), 469.

4. H. A. Bethe And J. Goldstone, Proc. Roy. Soc. (London) A238 (1957), 551.

5. G. Breit and W. G. Bouricius, Phys. Rev. 75 (1949), 1029; H. Feshbach and E. Lomon, Phys. Rev. 102 (1956), 891.

6. H. A. Bethe, Phys. Rev. 138 (1965), B804; R. Rajaraman and H. A. Bethe, Rev. Mod. Phys. 39 (1967), 745. 
7. L. D. Faddeev, Zh. Eksperim. Teor. Fiz. 39 (1960), 1459 (English transl.: Sov. Phys. (JETP) 12 (1961), 1014).

8. R. F. BISHOP, Phys. Rev. C 7 (1973), 479.

9. T. Hamada AND I. Johnston, Nucl. Phys. 34 (1962), 382.

10. R. V. ReID, Ann. Phys. (N. Y.) 50 (1968), 411.

11. H. Feshbach and E. Lomon, Phys. Rev. Lett. 6 (1961), 635.

12. R. F. Bishop, Ann. Phys. (N. Y.) 77 (1973), 106; also contains references to earlier literature.

13. R. Courant AND D. HilberT, "Methods of Mathematical Physics," Vol. 1, Interscience, New York, 1953.

14. R. OmNès, Nuovo Cimento 8 (1958), 316; N. I. Muskhelishvild, "Singular Integral Equations," P. Noordhoff, Groningen, Holland, 1953.

15. M. Razavy and D. W. Sprung, Phys. Rev. 133 (1964), B300; Y. E. Kim and A. Tubis, Phys. Rev. C 1 (1970), 414.

16. R. F. Bishop, M. R. Strayer, and J. M. Irvine, Phys, Rev. A 10 (1974), 2423. 